PNL-7632

UC-405

4c

\title{
Eigenanalysis of Large Electric Power Systems
}

D. M. Elwood

February 1991

Prepared for

the Bonneville Power Administration under a Related Services Agreement with the U.S. Department of Energy

Contract DE-AC06-76RLO 1830

Pacific Northwest Laboratory

Operated for the U.S. Department of Energy

by Battelle Memorial Institute 


\title{
DISCLAIMER
}

This repor was prepared as an account of work sponiored by an agency of the United States Government. Neither the United States Government nor any agency thereof, nor Battelte Memorial Institute, nor any of thair employees, makes any warranty, expressed or implied, or assumes any legal liability or responsibility for the accuracy, completeness, or usefulness of any inform ation, apparatus, product, or process disclosed, or represents that its use would not infringe privately owned rights. Reference herein to any specific commercial product, process, or service by trade name, trademark, manufacturer, or otherwise doe; not necessarily constitute or imply its endorsement, recommendation, or favoring by the United States Government or any agency thereof, or Battelle Memorial Institute. The views and opinions of authors expressed herein do not necessarily state or reilect those of the United States Government or any agency thereof.

\author{
PACIFIC NORTHWEST LABORA,TORY \\ operated by \\ BATTELLE MEMORIAL INSTITUTE \\ for the \\ UNITED STATES DEPARTMENT OF ENERGY \\ under Contract DE-ACO6-76RLO 1830
}

Printed in the United States of America

Available to DOE and DOE contractors irom the

Office of Scientific and Technical Information, P.O. Box 6,2, Oak Ridge, TN 37031;

prices available from (615) 576-8401. FTS 626-8401.

Available to the public from the National Technical Information Service, U.S. Department oi Commerce, 5245 Port Royal Rd., springfield, VA 22161. 


\title{
EIGENANALYSIS OF LARGE ELECTRIC
} POWER SYSTEMS

D. M. Elwood

February 1991

\author{
Prepared for \\ the Bonneville Power Administration \\ under a Related Services Agreement \\ with the U.S. Department of Energy \\ Contract DE-ACO6-76RLO 1830
}

Pacific Northwest Laboratory Richland, Washington 99352 

SUMMARY

Modern electric power systems are large and complicated, and, in many regions, the generation and transmission systems are operating near their limits. Eigenanalysis is one of the tools used to analyze the behavior of these systems. Standard eigenvalue methods require that simplified models be used for these analyses; however, these simplified models do not adequately model all of the characteristics of large power systems. Thus, new eigenanalysis methods that can analyze detailed power system models are required.

The primary objectives of the work described in this report were 1) to determine the availability of eigenanalysis algorithms that are better than methods currently being applied and that could be used on large power systems and 2) to determine if vector supercomputers could be used to significantiy increase the size of power systems that can be analyzed by a standard power system eigenanalysis code.

At the request of the Bonneville Power Administration, the Pacific Northwest Laboratory (PNL) conducted a literature review of methods currently used for the eigenanalysis of large electric power systems, as well as of general eigenanalysis algorithms that are applicable to large power systems. PNL found that a number of methods are currently being used for the this purpose, and all seem to work fairly well. Furthermore, most of the general eigenanalysis techniques that are applicable to power systems have been tried on these systems, and most seem to work fairly well. One of these techniques, a variation of the Arnoldi method, has been incorporated into a standard power system eigenanalysis package.

Overall, it appears that the general purpose eigenanalysis methods are more versatile than most of the other methods that have been used for power systems eigenanalysis. In addition, they are generally easier to use. For some problems, however, it appears that some of the other eigenanalysis methods may be better. Power systems eigenanalysis requires the computation of eigenvalues of nonsymmetric matrices. Such computations are fairly difficult, however, and they constitute an area of active research. Thus, 
research in this area should be closeiy monitored, and, as new methods become available, they should be tested on large power systems.

PNL also investigated the use of vector supercomputers to enlarge the size of power systems that can be analyzed by MASS, a standard power system eigenanalys is code. MASS was converted to run on a Cray supercomputer. On a conventional computer, MASS is limited to power system problems with about 500 states because of computer time constraints. Running MASS on a Cray X-MP $E A / 232$, however, PNL found that a problem with about 2200 states could be solved in about 26 minutes. Furthermore, by moving to a larger Cray, it should be possible to use MASS to analyze power systems with 5,000 to 10,000 states. Problems with 5,000 states would probably take about 5 cray hours, while a problem with 10,000 states would probably take about 43 Cray hours, though the actual execution times will depend on the type of cray used. Problems requiring 5 Cray hours are not uncommon. Problems requiring 43 Cray hours, however, would be fairly expensive. Thus, power systems with about 5000 states probably represent an upper limit on the size of problems that one would want to routinely solve using a Cray version of MASS. 


\section{CONTENTS}

SUMMARY $\ldots \ldots \ldots \ldots \ldots$

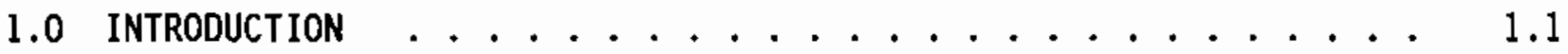

2.0 EIgENANALYSIS OF POWER SYSTEMS ............ 2.1

2.1 EIGENVALUES AND EIGENVECTORS . . . . . . . . . . 2.1

2.2 EIGENVECTORS AND DYNAMICAL LINEAR SYSTEMS . . . . . . 2.3

2.3 SUMMARY $\ldots \ldots \ldots \ldots . \ldots \ldots . \ldots . \ldots . \ldots . \ldots$

3.0 NUMERICAL MethodS FOR the EIgENVALUE PROBLEM $\ldots \ldots \ldots$

3.1 EIgENVALUES OF NONSYMMETRIC MATRICES .......... 3.1

3.2 EigenValues of LARGE PONER SYSTEMS . . . . . . . . 3.4

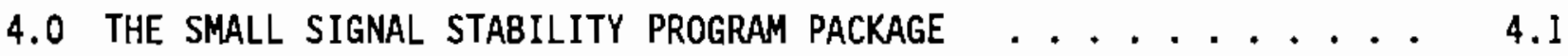

4.1 MASS ......................... 4.1

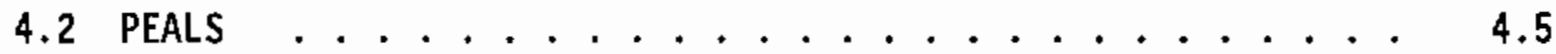

4.3 CONCLUSIONS $\ldots \ldots \ldots . \ldots . \ldots . \ldots . \ldots . \ldots$

5.0 MASS ON A CRAY SUPERCOMPUTER . . . . . . . . . . . . 5.1

5.1 VECTOR SUPERCOMPUTERS ....................... 5.1

5.2 MODIFICATION OF MASS ............... 5.3

5.3 VECTORIZATION OF MASS ............. 5.5

5.4 TESTING OF CMASS ............... . . . . . . .

5.5 PERfORMANCE OF MASS ON A CRAY . . . . . . . . . 5.11

5.6 CONCLUSIONS . . . . . . . . . . . . . 5.14

6.0 GRAPHICS AND EIGENANALYSIS . . . . . . . . . . . 6.1

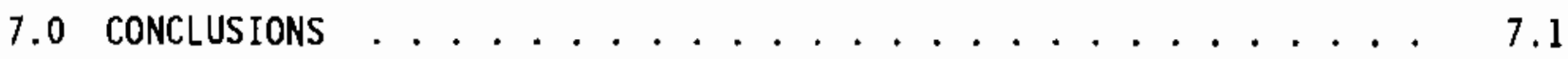

8.0 REFERENCES . . . . . . . . . . . . . . . . . 8.1 
APPENDIX A - CONVERSION OF MASS TO RUN ON A CRAY . . . . . . . . A.1

APPENDIX B - TESTING THE CRAY VERSION OF MASS . . . . . . . . B. B

APPENDIX C - A 2200-STATE CRAY VERSION OF MASS ......... . c.I 


\section{FIGURES}

5.1 Segmented Adder Operations . . . . . . . . . . . . 5.2

6.1 Generator Speed . . . . . . . . . . . . . 6.3

6.2 Generator Angle . . . . . . . . . . . . . . 6.4

\section{IABLES}

5.1 Execution Times (sec.) on a Cray X-MP/18 ......... 5.6

5.2 Speedup on a Cray X-MP/18 . . . . . . . . . . . 5.9

5.3 MASS Execution Times on Various Computers . . . . . . . 5.13 



\subsection{INTROOUCTION}

Modern electric power systems throughout the world are large and complex. In the United States, enormous amounts of power are transferred between various regions; in some of those regions, the generation and transmission systems are operating near their limits. The behavior of these large power systems is complicated. Thus, the reliable design and operation of a power system requires an understanding of this complex behavior and of the interactions between and among various parts of the system.

One method used in analyzing power system behavior is stability analysis. Two main types of power system stability are typically analyzed: 1) large signal stability and 2) small signal stability.

Large signal stability describes the response of a power system to large disturbances. The nonlinear characteristics of a power system have a significant impact on its response to large disturbances. Thus, the analys is of large signal stability involves using time domain methods to solve the nonlinear equations describing the behavior of the power system.

Small signal stability is the extent to which a power system responds to small disturbances. The nonlinear characteristics of the power system can usually be ignored when analyzing its response to small disturbances. Thus, small signal stability is usually analyzed by linearizing the system equations about a specific operating point and using linear analys is methods to analyze the Tinearized system.

In the past, large signal stability has been the main concern of power system analysts. However, as power systems increase in size and operate closer to their limits, sma11 signal stability is becoming more important. For example, actual cases of small signal instability have been observed in recent years (Van Ness et a1. 1980).

Currently available production-grade software can perform a complete eigenanalysis of power systems containing up to about 500 states (Kundur, Rogers, and Wong 1988). Modern power system problems, however, typically 
involve several thousand states. A variety of eigenanalysis methods is currently being tried for the analysis of these large power systems.

The Bonneville Power Administration (Bonneville) is interested in making eigenanalysis into a regular part of the power system design process. Thus, they commissioned the Pacific Northwest Laboratory (PNL) to study the problem of eigenanalysis of large power systems.

The work done for Bonneville consisted of three main tasks. The first task was to perform a literature review of methods that have been used for the eignenalysis of large power systems and of general eigenanalysis methods that could be applied to large power systems. The goals of this review were to determine the best methods currently available for the eigenanalysis of large power systems and to identify any new methods that might be used for this problem. The second task was to examine the factors limiting the size of problems that $c$ an be solved by a standard power system eigenanalysis package. The main part of this task consisted of converting one of the eigenanalysis programs in this package, MASS, to run on a Cray supercomputer. The objective here was to determine if modern supercomputers could be used to significantly increase the size of problems that can be solved using standard eigenanalysis methods. The third task was to examine ways of helping power system planners and analysts to interpret the results produced by an eigenanalysis code.

The report focuses primarily on describing 1) the results of the literature review and 2) the changes required to make MASS run efficiently on a Cray, including the results of test cases and a comparison of the performance of MASS on a Cray and on a conventional computer. This report also describes some simple types of graphics that can be used to help interpret the results produced by a power system eigenanalys is code.

This report contains several sections. In Section 2, the general technique of eigenanalysis is reviewed. Section 3 is a review of methods that have been used for the eigenanalys is of large power systems. Section 4 provides a look at the Small Signal Stability Program (SSSP) package (Kundur, Rogers, and Wong 1988) used for the eigenanalys is of power systems. In Section 5, the results of converting one of the SSSP programs to run on a Cray supercomputer are documented. Some graphics methods that could be used to 
help analyze the results obtained by an eigenanalysis program are described in Section 6. Finally, Section 7 provides a summary of the conclusions arrived at in the preceding six sections. Additional detailed material is provided in Appendixes A through $C$. 



\subsection{EIGENANALYSIS OF POWER SYSTEMS}

A tutorial review of the terminology and mathematics of eigenanalysis is provided in this section. The various quantities produced as output by a typical power system eigenanalysis code are described. Five quantities output by the eigenanalysis codes in the SSSP package are specifically defined and explained. These include 1) the state matrix, 2) eigenvalues, 3) eigenvectors, 4) participation factors, and 5) time and frequency responses. This review will be relatively brief because a number of good descriptions of eigenanalysis and power systems are available (Anderson, Agrawal, and Van Ness 1990; Hauer 1990; Kundur, Rogers, and Wong 1988).

The linearized equations describing the behavior of a power system can typically be written in the form

$$
\begin{aligned}
x^{\prime} & =A x+B u \\
y & =C x+D u
\end{aligned}
$$

where $x$ is a vector of states whose values describe the behavior of the system, $x^{\prime}$ denotes the time derivative of $x, u$ is the applied input to the system, $y$ is the output of the system, and matrices $A, B, C$, and $D$ are constant matrices. The A matrix is called the state matrix, and is of dimension $\mathrm{N}$ by $\mathrm{N}$, where $\mathrm{N}$ is the number of states of the system. One of the goals of power system eigenanalysis is to find the eigenvalues and eigenvectors of the state matrix. These quantities provide a great deal of information about the behavior of the system.

\subsection{EIGENVALUES AND EIGENVECTORS}

Every $\mathrm{N}$ by $\mathrm{N}$ matrix has $\mathrm{N}$ eigenvalues, $\lambda_{j}, j=1$ to $\mathrm{N}$, associated with it. These eigenvalues are the roots of the equation

$$
\operatorname{Det}(A-\lambda I)=0
$$


where $\mathrm{I}$ is the $\mathrm{N}$ by $\mathrm{N}$ identity matrix and Det is the determinant. The state matrix is real and nonsymetric; hence, the eigenvalues may be real or complex, with complex efgenvalues occurring in complex conjugate pairs. Some of the eigenvalues may be the same; i.e., Equation (2.2) may have multiple roots. For each eigenvalue, $\lambda_{j}$, there is at least one nonzero vector, $w_{i}$, which satisfies

$$
A w_{i}=\lambda_{j} w_{j}
$$

and at least one nonzero vector, $v_{i}$, which satisfies

$$
v_{\mathfrak{i}}^{\top} A=\lambda_{\mathfrak{j}} v_{\mathfrak{i}}^{\top}
$$

where $v_{j}^{\top}$ is the transpose of $v_{j}$. $w_{i}$ is called a right eigenvector, while $v_{j}$ is called a left eigenvector.

If the eigenvalues are distinct (i.e., $\lambda_{j} \lambda_{k}$ for $i k$ ), then there are $\mathrm{N}$ linearly independent right eigenvectors and $\mathrm{N}$ linearly independent left eigenvectors. Furthermore,

$$
\begin{aligned}
v_{j}^{\top} w_{k} & =d_{i}, \quad i=k, \\
& =0, \quad i \quad k
\end{aligned}
$$

where $d_{i}$ is a nonzero constant. $w_{i}$ and $v_{i}$ can be scated so that $d_{i}=1$. In the rest of this report, we assume that this scaling is used for the eigenvectors. The situation is significantly more complicated if there are repeated eigenvalues. In this report, all eigenvalues are assumed to be distinct; a discussion of the case when there are repeated eigenvalues is given in Wilkinson (1965).

Define $V$ and $W$ to be the matrices whose ith columns are equal to $v_{j}$ and $W_{j}$, respectively. Equation (2.5) implies that $V_{W}^{\top}=I$; hence,

$$
V^{\top}=W^{-1}
$$


Equation (2.3) can be written in matrix form to yield

$$
A W=W D
$$

where $D$ is a diagonal matrix whose $i$ th entry is equal to $\lambda_{i}$. Combining Equations (2.6) and (2.7) yields

$$
A=W D W^{-1}=W D V^{\top} \text {. }
$$

\subsection{EIGENVECTORS AND DYNAMICAL LINEAR SYSTEMS}

The results derived in Section 2.1 can be used to simplify the analysis of Equation (2.1). For simplicity, consider the linear system

$$
x^{\prime}=A x, \quad x(0)=x_{0}
$$

which is the same as Equation (2.1), except that $u=0$ and the output of the system has been ignored. Define a new set of variables, $y$, by

$$
y=V^{\top} x
$$

Substituting Equation (2.8) into Equation (2.9), multiplying Equation (2.9) by $V^{T}$, and then using Equations (2.6) and $(2.10)$, one finds that

$$
y^{\prime}=\text { Dy, } y(0)=V^{\top} x_{0} .
$$

However, $D$ is diagonal; hence, Equation (2.11) can be written

$$
y_{i}^{\prime}=\lambda_{i} y_{i}, \quad y_{i}(0)=v_{i}^{\top} x(0)
$$

The solution of Equation (2.12) is

$$
y_{i}=\exp \left(\lambda_{i} t\right) v_{i}^{T} x(0)
$$


Thus,

$$
y=\exp (D t) V^{\top} x(0)
$$

where $\exp (D t)$ is the diagonal matrix whose ith entry is equal to $\exp \left(\lambda_{i} t\right)$. Equations (2.6) and (2.10) imply that $x=$ Wy. Substituting this result into Equation (2.14) yields

$$
x(t)=W \exp (D t) v^{\top} x(0)=\sum_{i=1}^{N} w_{i} \exp \left(\lambda_{i} t\right) v_{i}^{\top} x(0)
$$

If $x(0)=w_{k}$, then Equation (2.15) reduces to $x(t)=w_{k} \exp \left(\lambda_{k} t\right)$.

Equation (2.15) shows that $x(t)$ can be written as the sum of terms whose time behavior is of the form $\exp \left(\lambda_{j} t\right)$. Thus, the functions $\exp \left(\lambda_{j} t\right), i=1$ to $\mathrm{N}, \mathrm{play}$ an important role in determining the behavior of the system defined by Equation (2.9). These functions will be called the modes of the system.

Equation (2.15) shows that 1 ) the $i$ th right eigenvector, $w_{j}$, determines how the $i$ th mode is distributed among the states of the system, i.e., $w_{i}$ determines the shape of the ith mode, and 2) the ith left eigenvector, via the term $v_{j}^{\top} \times(0)$, determines how strongly a particular initial condition, $x(0)$, excites the ith mode.

If $\lambda_{i}$ is real, then the ith mode is a real function. Furthermore, $w_{i}$ is real and the entries of $w_{j}$ determine the relative magnitude of the various states in the ith mode. There are three possible functional forms for the ith mode when $\lambda_{j}$ is real. First, if $\lambda_{j}=0$, then the mode is a constant function. Second, if $\lambda_{j}<0$, then the mode is an exponentially decaying function. Third, if $\lambda_{j}>0$, then the mode is an exponentially increasing function. Associated with $\lambda_{j}$ is the time constant $t_{c, j}=-I / \lambda_{j}$. The time constant is the amount of time required for the mode to decay by a factor of exp(1). If $\lambda_{j}>0$, then $t_{c, i}$ will be negative, indicating that the mode increases with time. 
If $\lambda_{j}$ is complex, then $\exp \left(\lambda_{j} t\right)$ is a complex sinusoid with amplitude $\exp \left(\operatorname{Re}\left(\lambda_{j}\right) t\right)$, where $\operatorname{Re}\left(\lambda_{j}\right)$ is the real part of $\lambda_{j}$. Furthermore, $w_{i}$ is complex, and the entries of $w_{i}$ determine the relative phases and magnitudes of the various states in the $i$ th mode. There are three possibie functional forms for the $i$ th mode when $\lambda_{j}$ is complex. First, if $\operatorname{Re}\left(\lambda_{j}\right)=0$, then the sinusoid has constant amplitude. Second, if $\operatorname{Re}\left(\lambda_{j}\right)<0$, then the sinusoid has an exponentially decaying amplitude. Third, if $\operatorname{Re}\left(\lambda_{j}\right)>0$, then the sinusoid has an exponentially increasing ampitude.

Several constants are associated with a complex $\lambda_{f}$. The time constant, $t_{c, j}=-1 / \operatorname{Re}\left(\lambda_{j}\right)$, is the amount of time required for the amplitude of the ith mode to decrease by a factor of $\exp (1)$. The frequency, $f_{j}=\left|\operatorname{Im}\left(\lambda_{j}\right)\right| / 2 \pi$, is the frequency of the $i$ th mode, where $\operatorname{Im}\left(\lambda_{i}\right)$ is the imaginary part of $\lambda_{j}$. Fina $] y$, the damping ratio, $\zeta_{j}=-\operatorname{Re}\left(\lambda_{j}\right) /\left(\left[\operatorname{Im}\left(\lambda_{j}\right)\right]^{2}+\left[\operatorname{Re}\left(\lambda_{j}\right)\right]^{2}\right)$, is a nondimensional measure of stability. A value of $\zeta_{j}$ near +1 indicates that the amplitude of the mode decreases rapidly, relative to the period of the oscillations, while a small positive value indicates that the amplitude of the mode decreases slowly, relative to the period of the oscillations. A negative value for $5_{i}$ indicates that the amplitude of the mode increases with time.

The preceding discussion shows that if the real part of any eigenvalue is positive, then the mode corresponding to that eigenvalue grows exponentially with time. This is clearly an undesirable property in a power system, and it is useful to know when undesirable modes can be controlled.

The method used to analyze Equation (2.9) can be applied to Equation (2.1). The result of such an anaTysis (Kundur, Rogers, and Wong 1988) yietds

$$
\begin{aligned}
x(t) & =W \exp (D t) v^{\top} x(0)+\int_{0}^{t} W \exp (D(t-s)) v^{\top} B u(s) d s \\
& =\sum_{i=1}^{N} w_{i}\left[\exp \left(\lambda_{i} t\right) v_{i}^{\top} x(0)+\int_{0}^{t} \exp \left(\lambda_{j}(t-s)\right)\left(v_{i}^{\top} B\right) u(s) d s\right],
\end{aligned}
$$




$$
\begin{aligned}
y(t)= & \sum_{i=1}^{N} c w_{i}\left[\exp \left(\lambda_{i} t\right) v_{i}^{\top} x(0)+\int_{0}^{t} \exp \left(\lambda_{j}(t-s)\right)\left(v_{i}^{\top} B\right) u(s) d s\right] \\
& +D u(t) .
\end{aligned}
$$

The first term in Equation (2.16) represents the natural response of the system to the initial conditions, and is the same as $x(t)$ in Equation (2.15). The second term represents the response of the system to the input, $u(t)$. Equation (2.16) shows that if $v_{i}^{\top} B=0$, then an input, $u(t)$, will have no effect on the ith mode. If this happens, then the ith mode is uncontrollable. Equation (2.17) shows that if $\mathrm{Cw}_{j}=0$, then the $i$ th mode has no effect on $y(t)$. If this happens, then the ith mode is unobservable.

It is useful to know how the system defined by Equation (2.1) responds to various inputs. Some responses usually of interest are 1) the time response to a step or impulse input and 2) the steady-state frequency response to a sinusoidal input, $\sin (\omega t)$. A1l of these responses can be computed using Equation (2.17). The programs in the SSSP package allow these types of responses to be computed; the results are output in both tabular format and plot format. If frequency responses are computed, then the transfer functions, i.e., the responses divided by the input, are also printed.

When trying to control undesirable modes, it is useful to know how sensitive the eigenvalues are to changes in the values of the elements of the state matrix. The eigenvectors allow these sensitivities to be computed. In particular, one finds (Rogers and Kundur 1990) that

$$
\partial \lambda_{i} / \partial a_{j, k}=v_{i}^{\top} \partial A / \partial a_{j, k} w_{i}=v_{i, j} w_{i, k}
$$

where $a_{j, k}$ is the entry in the jth row and kth column of $A, v_{i, j}$ is the $j$ th element in $v_{i}$, and $w_{i, k}$ is the kth element in $w_{i}$.

The participation vector, $p_{j}$, corresponding to eigenvalue $\lambda_{i}$ is defined to be

$$
p_{j, k}=v_{i, k} w_{i, k}
$$


where $p_{i, k}$ is the kth entry in $p_{j}$ (Perez-Ariaga, Verghese, and Schweppe 1982). Thus, the participation vectors represent the sensitivities of the eigenvalues to perturbations in the diagonal entries of the state matrix. The elements of the participation vectors are dimensionless variables, so are independent of the units used for the state variables. The relative magnitudes of the entries in an eigenvector, however, depend upon the system of units used for the state variables. Thus, the magnitudes of the elements in the ith participation vector are frequently used to determine which states are most important in the ith mode (Rogers and Kundur 1990). The MASS program in the SSSP package defines the dominant state for the ith mode to be the state corresponding to the element of $p_{i}$ having the largest magnitude and prints the dominant states along with the eigenvalues.

\subsection{SUMMARY}

This chapter has reviewed the major elements of eigenanalysis. The main results obtained from eigenanalysis are 1) eigenvalues, 2) right eigenvectors, and 3) left eigenvectors. The eigenvalues determine the time behavior, i.e., frequency and rate of decay, of the naturat modes of a system. The right eigenvectors determine the shape of a mode. The left eigenvectors determine the extent to which a particular initial condition excites a mode. The eigenvalues and eigenvectors can also be used to compute the time response and frequency response of a system to various inputs. Furthermore, the eigenvectors are used to determine the sensitivities of the eigenvalues to perturbations in the elements of the state matrix. The vector containing the sensitivities of an eigenvalue to perturbations in the diagonal entries of the state matrix is called a participation vector; it is sometimes used as a measure of the relative importance of the states to a given mode. 



\subsection{NUMERICAL METHODS FOR THE EIGENVALUE PROBLEM}

A number of programs (Dandeno and Kundur 1976; Gross, Imparato, and Look 1982; Undri11 1968) are available that form the state matrix of a power system and compute its eigenvalues and eigenvectors using the $Q R$ algorithm. The $Q R$ a1gorithm (Wilkinson 1965) is the standard method used to compute the eigenvalues and eigenvectors of small to moderately sized problems, but computer time and memory constraints usually limit its use to problems with about 500 states. Typical power systems, however, are at least an order of magnitude larger than this. Hence, simplifications must be made before they can be analyzed using programs based on the QR algorithm. For this reason, recent work has focused on finding methods that can be used to find some of the eigenvalues of these large power systems but without making simplifying assumptions. None of these newer methods actually forms the state matrix.

Rogers (1987) reviewed eigenvalue methods that had been used on large power systems up to 1987 . This section will briefly review 1) the main methods used to compute the eigenvalues of nonsymmetric matrices, 2) the results in Rogers (1987), and 3) work that has been done on large power systems since 1987.

\subsection{EIGENVALUES OF NONSYMMETRIC MATRICES}

The eigenvalues of symmetric matrices are always real and well conditioned; i.e., small perturbations in the matrix produce smal1 perturbations in the eigenvalues. The eigenvalues of nonsymmetric matrices, however, are generally complex and can be 111 -conditioned; i.e., small changes in the matrix may produce large changes in some of the eigenvalues (Wilkinson 1965). Thus, it is much easier to compute eigenvalues of symmetric matrices than it is to compute eigenvalues of nonsymmetric matrices. Furthermore, many good methods are currently available to compute some of the eigenvalues of large, sparse, symmetric matrices (Cullum and Willoughby 1985; Parlett 1980, 1984). A number of methods are also available for computing eigenvalues of large, sparse, nonsymmetric matrices (Kerner 1989), but these methods are not as we 11 understood as the algorithms for symmetric matrices. 
The main method used to compute the eigenvalues of small to medium sized matrices is the QR algorithm (Wilkinson 1965; Smith et al. 1976). It can be shown (Golub and Van Loan 1983) that the eigenvalues computed by the $Q R$ algorithm are the eigenvalues of a matrix $A+E$, where $A$ is the matrix whose eigenvalues are required and $E$ is a matrix of small perturbations. Thus, well conditioned eigenvalues will be computed fairly accurately, while i11conditioned eigenvalues may be computed inaccurately. Finally, the QR algorithm does not take advantage of sparsity in matrices, so it is not practical for computing the eigenvalues of very large, sparse matrices.

The main methods used to compute eigenvalues of large, sparse, nonsymmetric matrices are variations of 1) the power method, 2) the Lanczos algorithm, and 3) the Arnoldi algorithm, and will be described below. These algorithms generally require that the product $A x$ be computed, but do not actually modify $A$, where $x$ is an arbitrary vector and $A$ is the matrix whose eigenvalues are required. Thus, sparse matrix methods can be used to compute $A x$. In the following, $A$ will denote a general $N$ by $N$ matrix with eigenvalues $\lambda_{j}, i=1$ to $N$. It is assumed that the eigenvalues are ordered so that $\left|\lambda_{j}\right| \geq\left|\lambda_{i+1}\right|, i=1$ to $N$; hence, $\lambda_{1}$ is the dominant eigenvalue of $\mathrm{A}$.

The power method (Wilkinson 1965) forms the sequence of vectors

$$
\begin{aligned}
& y_{k+1}=A x_{k}, k=0,1,2, \ldots \\
& \lambda^{k}=y_{k, j}, \text { where }\left|y_{k, j}\right|=\operatorname{MAX}\left|y_{k, j}\right| \\
& x_{k+1}=y_{k} / \lambda^{k}
\end{aligned}
$$

where $x_{0}$ is an arbitrary starting vector and $y_{k, i}$ is the ith entry of $y_{k}$. In general, $\lambda^{k}$ converges to $\lambda_{1}$ (the dominant eigenvalue of $A$ ) and $x_{k}$ converges to the eigenvector corresponding to $\lambda_{1}$. The power method has the disadvantage that it can be used to find only the dominant eigenvalue of $A$. The power method can be used with deflation (Wilkinson 1965), however, to find a few of the dominant eigenvalues of a matrix. Finally, simultaneous iteration (Jennings and Stewart 1975; Stewart and Jennings 1981) can be used to find 
several of the dominant eigenvalues and corresponding eigenvectors of a matrix. Simultaneous iteration is an extension of the power method that performs iterations using several vectors rather than just one.

The Lanczos algorithm (Wilkinson 1965) generates a sequence of tridiagonal matrices, $T_{m}$, such that, as $m$ increases, some of the eigenvalues of $T_{m}$ become better approximations to some of the eigenvalues of $A$. The standard Lanczos algorithm is characterized by a number of difficulties, however. Thus, modified versions of the Lanczos algorithm exist that 1) do no reorthogonalization (Cullum and Willoughby 1984; CuTlum, Kerner, and Willoughby 1989), or 2) use a "look-ahead" algorithm (Parlett, Taylor, and Liu 1985) to overcome some of the difficulties with the standard Lanczos algorithm.

The Arnoldi algorithm (Wilkinson 1965) is similar to the Lanczos algorithm, but generates a sequence of upper Hessenburg matrices, $H_{m}$, some of whose eigenvalues are approximations to the eigenvalues of $A$. An upper Hessenburg matrix, $H$, is a matrix such that $h_{i, j}=0$ for $i>j+1$. The standard Arnoldi algorithm, however, requires a lot of storage and suffers numerical problems. Thus, an iterative Arnoldi algorithm with reorthogonalization (Saad 1980) has been developed and seems to work fairly well. Furthermore, acceleration techniques (Saad 1984; Ho 1990) have been developed to speed up the convergence of the Arnoldi algorithm. The standard Arnoldi algorithm does not give approximations to the left eigenvectors of a matrix. Thus, a two-sided Arnoldi algorithm (Ruhe 1983) has been developed. It appears, however, that only a limited amount of work has been done on this algorithm. Furthermore, it is possible to apply the Arnoldi algorithm to $A^{\top}$ to find the left eigenvectors of $A$, and it is unknown whether this is better than using the two-sided Arnoldi algorithm. Finally, Sorensen (1990) gives another variation of the Arnoldi algorithm.

A11 of the sparse eigenvalue methods described above tend to find the dominant eigenvalues of $A$. Frequentiy, however, one wants to find the eigenvalues in a particular region of the complex plane, not the dominant eigenvalue. This is not a problem, because all of the methods described above can be used with a "shift and invert" strategy to find the eigenvalues nearest a particular shift. In the shift and invert strategy, 1) a shift, s, is 
selected and 2) the eigenvalues of $(A-s I)^{-1}$ are computed, where $I$ is the $N$ by $N$ identity matrix. The eigenvalues of $(A-s I)^{-1}$ are $1 /\left(\lambda_{j}-s\right)$; hence, the dominant eigenvalues of this matrix correspond to the eigenvalues of $A$ that are closest to the shift point, $s$. The shift and invert strategy, and other algorithms, are described by Saad (1989).

Currently, there does not appear to be a best method of computing eigenvalues of large, sparse, nonsymmetric matrices, and much research is now being done on this topic. Simultaneous iteration, variations of the Lanczos algorithm, and variations of the Arnoldi algorithm, however, appear to work well in many cases. Simultaneous iteration is a fairly straightforward algorithm, but 1) can be slow to converge and 2) is generally able to find only a few eigenvalues, and corresponding eigenvectors, at a time. The Arnoldi algorithm seems to be faster than simultaneous iteration, but is also limited in the number of eigenvalues and eigenvectors that it can find at one time. The Lanczos algorithm of Cullum and Willoughby (1984) has the potential of computing a large number of eigenvalues and eigenvectors of large matrices, but is less well understood than some of the other algorithms. Furthermore, the Lanczos algorithms require the use of both $A$ and $A^{\top}$. Finaliy, the possibility of $i 11$-conditioned eigenvalues suggests that it would be a good idea to compute error bounds for the eigenvalues of nonsymmetric matrices, regardless of what method is used to compute the eigenvalues. A method for computing error bounds is given in Kahan, Parlett, and Jiang (1982).

\subsection{EIGENYALUES OF LARGE POWER SYSTEMS}

The Tinearized state equations for a power system can typically be written

$$
\begin{aligned}
& x^{\prime}=J_{A} x+J_{B} V \\
& 0=J_{C} x+J_{D} V
\end{aligned}
$$


where $x$ is the vector of state variables, $x^{\prime}$ is the time derivative of $x, V$ is the vector of system voltages, and $J_{A}, J_{B}, J_{C}$, and $J_{D}$ are sparse matrices that depend on the system parameters. The system voltages can be eliminated from Equation (3.2) to yield

$$
x^{\prime}=A x
$$

where $A=J_{A}-J_{B} J^{-1} J_{C}$ is the system state matrix. The state matrix is not sparse (Semlyen and Wang 1988) and is generally large, real, and nonsymmetric. The eigenvalues of power systems with up to about 500 states can easily be computed by forming the state matrix and using the QR algorithm to compute its eigenvalues. For very large power systems, however, this is impractical because of computer time and memory constraints. Thus, various methods that compute the eigenvalues of the power system without forming the state matrix have been tried. Most of these methods use some form of one of the sparse matrix algorithms described in section 3.1 and compute the product Ax indirectly by using the sparse matrices $\mathrm{J}_{A}, \mathrm{~J}_{B}, \mathrm{~J}_{C}, \mathrm{~J}_{D}$.

The Analysis of Essentially Spontaneous Oscillations in Power Systems (AESOPS) algorithm (Byerly, Bennon, and Sherman 1982; Rogers 1987) requires the user to supply an estimated eigenvalue and to specify a generator in the power system. The program takes the estimated eigenvalue and 1) applies a torque, which is a function of the estimated eigenvalue, to the specified generator, 2) computes the response of the linear system to the torque, and 3) computes a new estimate for the eigenvalue based on the response of the linear system. This is repeated until convergence occurs. The equations used by AESOPS are approximations that were derived from the equations for the eigenvalues of a single classical generator and infinite bus. The Program for Eigenvalue Analysis of Large Systems (PEALS) (Wong et al. 1988) is similar to AESOPS, but PEALS retains more of the sparsity of the problem; hence, it is more efficient than AESOPS. PEALS requires that good starting estimates be available for the desired eigenvalues, so will be of 1 imited use when this information is unavailable. 
The $S$ matrix method (Uchida and Nagao 1988) computes some of the eigenvalues and eigenvectors of the matrix $S=(A+h I)(A-h I)^{-1}$, where $h$ is a positive number, using the power method or an iterative, block version of the Lanczos algorithm. If the eigenvalues of $A$ are $\lambda_{j}, i=1$ to $N$, then the eigenvalues of $S$ are given by $\left(\lambda_{j}+h\right) /\left(\lambda_{i}-h\right)$. Thus, the eigenvalues of $A$ that are in the right half-plane are mapped into eigenvalues of $S$ that are contained in the unit disk, while eigenvalues of $A$ in the left half-plane are mapped into eigenvalues of $S$ that are outside the unit disk. The net effect of this transformation is that the eigenvalues of $A$ that correspond to unstable modes are transformed into the dominant eigenvalues of $S$. The power method was discussed in Section 3.1. The block Lanczos algorithm used with the $S$ matrix method is an extension of the standard Lanczos algorithm that works with blocks of two vectors rather than single vectors. The use of the $S$ matrix is useful for finding unstable modes, but there does not appear to be any reason to think that the block Lanczos algorithm used with the $S$ matrix is superior to other algorithms for finding the eigenvalues of the $S$ matrix.

Martins (1986) uses inverse iteration to find the eigenvalue and corresponding eigenvector closest to a specified point in the complex plane. The computations utilize the sparse matrices $J_{A}, J_{B}, J_{C}$, and $J_{D}$ instead of the actual state matrix. This algorithm suffers from the fact that reasonable estimates of the desired eigenvalues must be available before it can be used.

SemTyen and Wang (1988) compute some of the eigenvalues of a power system in two stages. The first stage consists of 1) using initial estimates of some eigenvalues to simplify the power system and 2) computing all of the eigenvalues for the reduced system. The second stage consists of using inverse iteration, Newton's method, and deflation to refine the eigenvalues obtained in the first stage.

Selective modal ana7ysis (Perez-Arriaga, Verghese, and Schweppe 1982) uses a reduced set of equations and an iterative technique to find some of the eigenvalues of the unreduced system.

Wang and Semlyen (1989) applied simultaneous iteration (Stewart and Jennings 1981) and the iterative Arnoldi algorithm (Saad 1980) to power system problems and compared the results with some of the methods described above. 
They found that 1) the Arnoldi algorithm was faster than simultaneous iteration but required more memory; 2) simuttaneous iteration was significantly faster than inverse iteration when three eigenvalues were found; and 3) a version of the Lanczos algorithm in Uchida and Nagao (1988) was used but did not converge. They concluded that 1) simultaneous iteration and the Arnoldi algorithm were more reliable and efficient than the other two algorithms; and 2) for very large problems, simultaneous iteration would probably be superior to the Arnoldi algorithm because of storage requirements. Another advantage of the Arnoldi algorithm and simultaneous iteration is that they can be used with a search algorithm to find eigenvalues in a specific region of the complex plane, without any previous knowledge of where the eigenvalues might be located.

Finally, Van Ness and Boratynska-Stadnicka (1990) describe a method in which 1) the power system is divided into subsystems, 2) the eigenvalues of the subsystems are obtained using standard eigenvalues programs, and 3) various methods are used to estimate the changes in the eigenvalues that occur as a resuit of interactions between the subsystems. This algorithm is well suited to parallel computing and has been implemented on a hypercube computer.

Many of the algorithms described in this section require initial estimates for the desired eigenvalues. For problems where this information is available, these algorithms seem to work. Algorithms such as simultaneous iteration and the Arnoldi algorithm, however, can be used when these estimates are not available, and are fairly easy to use. Overall, it seems that general purpose algorithms such as simultaneous iteration, variations of the Arnoldi algorithm, and variations of the Lanczos algorithm provide the most flexibility for finding some of the eigenvalues of a large power system. Some of the other algorithms described here, however, may be more useful for some problems. Finally, we note that the task of computing eigenvalues of nonsymmetric matrices is difficult, and programs designed to do this must be wel1 designed and tested. 



\subsection{THE SMALL SIGMAL STABILITY PROGRAM PACKAGE}

The Sma11 Signa1 Stability Program (SSSP) package (Kundur, Rogers, and Wong 1988; Rogers and Kundur 1990) consists of two eigenvalue programs. The MultiArea Small Signal (MASS) stability program is designed to calculate all of the eigenvalues of power systems with up to about 500 states, while the Program for Eigenvalue Analysis of Large Systems (PEALS) is designed to calculate the eigenvalues of very large power systems. In this section, 1) the factors limiting the size of problem that can be solved by MASS and PEALS are described; 2) ways of increasing the problem size that these codes can solve are considered; and 3 ) modifications that would improve the two codes are described.

Two test problems, the T3500 and BCH88HW3 3 problems, were used to evaluate MASS and PEALS and are referred to later in this section. The T3500 problem has 467 states, while the BCH88HW3_3 problem has 2165 states. These problems are described in more detail in Section 5.

\subsection{MASS}

MASS computes eigenva]ues and right eigenvectors of a matrix using the $Q R$ a] gorithm from EISPACK (Smith et al. 1976). It computes the left eigenvectors by inverting the matrix of right eigenvectors [see Equation (2.6)]. The $Q R$ algorithm, discussed in Section 3 , is the standard method used to compute the eigenvalues and eigenvectors of small to medium sized systems.

MASS inverts the matrix of right eigenvectors by solving the linear systems

$$
W x_{i}=e_{i}, \quad i=1, N
$$

for $x_{i}$, where $W$ is the matrix of right eigenvectors, $N$ is the number of states, the ith entry of $e_{j}$ is equal to one, and all other entries of $e_{j}$ are equal to zero. $x_{i}$ is the $i$ th column of $w^{-1}=V^{\top}$, where the kth row of $V^{\top}$ is the kth left eigenvector. MASS solves Equation (4.1) using Gaussian elimination with partial pivoting (Golub and Van Loan 1983). Gaussian elimination 
with partial pivoting is the standard method of solving linear systems. The accuracy of the results, however, depend on the condition number of $W$ (Dongarra et a1. 1979, Chapt. 1; Golub and Van Loan 1983, pp. 71-78). As the condition number of $W$ increases, the number of correct digits in the computed value of $x_{j}$ usually decreases. The current version of MASS does not compute and print the condition number of $W$. The condition number can be estimated fairly easily and rapidly (Dongarra et al. 1979), and it is recomnended that MASS be modified to do this. This would give an indication of the accuracy of the left eigenvectors.

The 4000-bus VAX version of MASS requires about two megawords of memory, but would require approximately $4 N^{2}+1,202,000$ words of memory, where $N$ is the number of states in the probTem, for $N$ greater than about 1000 . The EISPACK subroutine HQR2 is used by MASS to compute eigenvalues and right eigenvectors, and uses two $N$ by $N$ arrays. This requires $2 N^{2}$ words of memory. The Teft eigenvectors and participation factors can be computed using the arrays that were used by subroutine HQR2, though the current version of MASS uses a third $\mathrm{N}$ by $\mathrm{N}$ array. Finally, the time response and frequency response calculations could be done using one of the arrays that was used by subroutine HQR2, but MASS actualiy uses a fourth $N$ by $N$ array. Thus, the portions of MASS that compute the eigenvalues, eigenvectors, participation vectors, time response, and frequency response require a total of about $4 \mathrm{~N}^{2}$ words of memory.

It appears that it would be fairly simple to modify these sections of MASS so that only $2 \mathrm{~N}^{2}$ words of memory are required. The sections of MASS that assemble the state matrix, however, appear to use parts of three different $N$ by $\mathrm{N}$ arrays, and it may be fairly difficult to reduce the memory requirements of these code sections. Given a fixed amount of memory, a version of MASS that requires only $2 N^{2}+1,202,000$ words of memory would be able to solve a problem about 40 percent larger than a version of MASS requiring $4 N^{2}+1,202,000$ words of memory. Thus, modifying MASS to reduce its memory requirements would be worthwhile only if 1 ) it is being run on a machine where memory, not computer time, is the main factor limiting the size of problem that can be solved and 2) a 40-percent increase in problem size is considered to be worth the effort of modifying MASS. 
The total execution time required to do an eigenanalys is of the $T 3500$ problem using MASS is about 115 minutes on a MicroVAX and about 36 minutes on a VAX 8650. Detailed timing data on the MicrovaX shows that the execution time of MASS can be broken down as follows: 1) about 70 percent of the time is used to compute the eigenvalues and right eigenvectors; 2) about 24 percent of the time is used to invert the matrix of right eigenvectors; and 3) about 6 percent of the time is used to do input, output, and to generate the state matrix. MASS uses what is generally considered to be the best software currently available for computing all of the eigenvalues and right eigenvectors of a small to moderately sized matrix. Consequently, there do not appear to be any ways to significantly reduce the execution time of this portion of MASS. The algorithm used by MASS to invert the right eigenvector matrix (i.e., compute the left eigenvectors) is fairly standard. However, it does not take advantage of the special form of the vectors $e_{j}$ in Equation (4.1). Modifying the matrix inversion section of MASS to take advantage of the special form of the $e_{j}$ would moderately reduce the execution time of this section of MASS, but would probabiy reduce the total execution time of MASS by less than 10 percent. Thus, although this modification would be easy to make, it would not significantly increase the size of problem that can be solved using a given amount of computer time.

The execution times of the aTgorithms used to compute eigenvalues and eigenvectors are proportional to $\mathrm{N}^{3}$. Thus, a 1000 -state problem would take about 6 hours to run on a VAX 8650 , given that the 467 -state $T 3500$ problem takes about 36 minutes; and a 2000-state problem would take about 48 hours. These results ignore the effects of page faults that could significantly increase the actual execution times for the 1000- and 2000-state problems. These estimated execution times indicate that computer time will probabiy 1 imit the use of MASS, on computers comparable to the VAX 8650 , to problems with 500 to 1000 states. The current version of MASS would require about 5 million words of memory for a problem with 1000 states. This can be handled by most modern computers. Thus, the main factor limiting the size of problem that can be solved by MASS, on computers comparable to the VAX 8650 , will tend to be computer time, not memory. Hence, modifying MASS to reduce its memory requirements would be of 1 imited value. 
The current version of MASS always computes and outputs the eigenvalues and the dominant state associated with each eigenvalue. Determining the dominant states for the eigenvalues requires that the right and left eigenvectors and the participation factors be computed. Thus, these quantities are a Tways computed by MASS. If the user wants to know the eigenvalues but not the dominant states, eigenvectors, or other features, then MASS does significantly more work than required. In fact, EISPACK has a subroutine, HQR, that computes eigenvalues only and is about twice as fast as subroutine HQR2. Thus, the total execution time of MASS could be reduced by about 50 percent for those problems in which only the eigenvalues are required. Furthermore, in many problems the eigenvectors are needed for only a limited set of the eigenvalues. In this case it is possible to use subroutine HQR to compute the eigenvalues and then use the inverse power method to computed the required eigenvectors. EISPACK has subroutines for computing right eigenvectors using the inverse power method, and these could be modified to compute left eigenvectors. Overall, this modification would probably reduce the execution of MASS by about 50 percent or less. Reducing the execution time of MASS by 50 percent would increase the size of problem that could be solved, using a given amount of computer time, by only about 25 percent. Thus, making the modifications described above would be of 1 imited value, but would be fairly easy to do.

The preceding results show that MASS can be used to solve problems with about 1000 states on computers comparable to the VAX 8650 . Furthermore, the algorithms in MASS are fairly efficient; there do not appear to be any software improvements that would significantly increase the size of problem solvable by MASS. Thus, the only way that MASS could be used to solve a problem with significantly more than 1000 states is to run it on a computer that is significantly faster than the $V A X 8650$. It was previousiy estimated that MASS could solve a 1000-state problem on a VAX 8650 in about 6 hours. This implies that MASS would have to run 8 times faster to solve a 2000-state problem in 6 hours, and 64 times faster to solve a problem with 4000 states in 6 hours. This indicates that for MASS to solve problems with several thousand states, in a reasonable amount of time, it would have to be run on a machine that is at least 50 to 100 times faster than a $V_{A X} 8650$. Note that al though 
a specific machine may be 50 times faster than a VAX 8650 , MASS may run only 5 or 10 times faster on that machine than it does on a VAX 8650 .

There are essentially two classes of high-performance computers on which MASS could be run. Vector supercomputers, such as a Cray, are probably the best option, while parallel supercomputers, such as the Intel iPSC/860, are another option. Vector supercomputers are likely to be the best for running MASS because 1) the main computational work in MASS is linear algebra; 2) vector supercomputers are very good at doing most linear algebra computations; and 3) it is fairly easy to modify most FORTRAN codes, at least those using standard FORTRAN 77, to run efficiently on a vector supercomputer.

Parallel supercomputers have the potential to solve very large problems, but attempts to modify the $Q R$ algorithm to run efficiently on parallel computers have had limited success (Boley, Maier, and Kim 1989; Geist and Davis 1990).

MASS outputs all results (eigenvalues, eigenvectors, and others) in the form of tables and/or plots and outputs only a few significant digits. It would be useful if an option were added to MASS to allow the user to specify that some, or all, of these quantities be printed to a "dump" file from which they could be retrieved by other programs. This would make it easier to develop and use programs to analyze the resuTts obtained by MASS.

The information reviewed in this section indicates that 1) MASS is a good software package for performing the eigenanalysis of power systems with up to 500 to 1000 states and 2) the only means of significantly increasing the size of problem that can be solved by MASS is to run it on a supercomputer. It would be useful, however, if MASS were modified so that it computes and prints the condition number of the matrix of right eigenvectors and outputs the eigenva7ues, eigenvectors, and so on to a "dump" file.

\subsection{PEALS}

The PEALS program was designed to compute selected eigenvalues of systems that are too large to be analyzed using MASS. PEALS computes eigenvalues using either the modified AESOPS (PEALS) algorithm (Wong et a1. 1988) or the iterative Arnoldi algorithm (Wang and Semlyen 1989). Both algorithms were discussed in Section 3. 
The large VAX version of PEALS is dimensioned for 12,000 buses, 30,000 lines, 2200 devices, and 22,000 states, and requires about four megawords of memory. Thus, the memory requirements do not appear to be a significant factor in limiting the types of problems that PEALS can solve.

The Arnoldi algorithm was used on a Microvax to find 10 eigenvalues of the $T 3500$ problem near the shift point $0+j 6$. The AESOPS algorithm was then used to find the same eigenvalues. The first two significant digits of the Arnoldi results were used as the initial eigenvalue estimates for the AESOPS algorithm. For each eigenvalue, the generator with the largest participation factor, as computed by the Arnotdi method, was used as the disturbed generator. The Arnoldi algorithm required about 30 minutes to find the 10 eigenvalues and corresponding right and left eigenvectors, while the AESOPS algorithm required about 87 minutes. The preceding conditions were repeated for the BCH88HW3 3 test problem, but with two changes: 1) the shift point was $0+j 4.2$ and 2) the AESOPS algorithm was used to compute only the first three eigenvalues found by the Arnoldi algorithm. The Arnoldi algorithm required approximately 40 minutes to find the 10 eigenvalues and eigenvectors, while the timing data show that the AESOPS algorithm would have taken about 107 minutes to find a 1110 eigenvalues and eigenvectors. The AESOPS time was computed using the average time taken to find three of the eigenvalues and corresponding eigenvectors. In all of these cases, the differences between the eigenvalues computed using the Arnoldi algorithm and the AESOPS algorithm were less than 0.0001 . The 10 eigenvalues nearest the shift $0+j 6$ were extracted from the results computed by MASS for the $T 3500$ problem and were found to differ from the results computed using the Arnoldi algorithm by less than 0.0001 . Finaliy, the 10 eigenvalues nearest the shift $0+j 4.2$ were extracted from the results computed by the Cray version of MASS (see Section 5) for the BCH88HW_3 problem and were found to differ from the results computed by the Arnoidi algorithm by less than 0.006 . This is not unreasonable, because the Cray uses 64-bit arithmetic while the VAX uses 32-bit arithmetic. In both cases, the Arnoldi algorithm correctly found the 10 eigenvalues closest to the user-specified shift. These results indicate 
that both the AESOPS and Arnoldi algorithms in PEALS give essentialiy the same results, but that the Arnoldi algorithm is almost 3 times faster than the AESOPS algorithm.

For most problems, it appears that the Arnoldi algorithm in PEALS is superior to the AESOPS algorithm. The reasons for this are 1) the Arnoldi algorithm is significantly faster than the AESOPS algorithms, at least on the problems considered above; 2) the AESOPS algorithm can find eigenvalues associated onfy with rotor angle modes, while the Arnoldi algorithm does not have this restriction; and 3 ) the user must know a fair amount about the system being studied, i.e., must have estimates for the eigenvalues and must know which generators to disturb, to use the AESOPS algorithm, while the Arnoldi algorithm does not require this knowledge.

The timing data from the preceding problems showed that, for both the Arnoldi and AESOPS algorithms, 1) input and output represent a fairly smal1 fraction of the execution time of PEALS and 2) the sparse matrix subroutines use a significant fraction of the execution time of PEALS. Because PEALS was designed to efficiently handle the sparse matrix equations found in power systems (Wong et al. 1988), it would probably be fairly difficult to reduce the code's execution times for those matrix algorithms. No other obvious software modifications would significantiy decrease the execution time of PEALS.

Ten eigenvalues of the $T 3500$ and $\mathrm{BCH} 88 \mathrm{HW} 33$ problems were found using the Arnoldi algorithm on a VAX 8650 and took 5 to 10 minutes to run. Furthermore, the execution time of the Arnoldi algorithm appears to increase fairly slowly as the problem size increases. These results indicate that a few of the eigenvalues of large power systems can be found relatively quickly using the Arnoldi algorithm on a computer comparable to the VAX 8650. If eigenvalues near a few dozen shifts are required, however, the Arnoldi algorithm will probably take several hours to run on a VAX 8650 , and it may be worth running PEALS on a faster machine.

The Arnoldi algorithm in PEALS requires the user to specify 1) a shift point, 2) the number, $m$, of eigenvalues desired, and 3) a set of error tolerances. PEALS then computes m numbers, which are estimates of $m$ of the 
eigenvalues near the specified shift. The PEALS output indicates which eigenvalues converged and which did not converge, and gives an iteration sumnary showing the residuals for each eigenvalue on each iteration. PEALS allows the eigenvalues near several different shift points to be computed in one run using "change case" data. Thus, estimates for eigenvalues in a region of the complex plane can found by using a set of shift points that are distributed throughout the region of interest. This search strategy is described in more detail by Wang and Semlyen (1989). The Arnoldi algorithm in PEALS would be easier to use if the user could specify a region of the complex $p l$ ane and have PEALS automatically search that region for eigenvalues.

Like MASS, PEALS outputs all results in the form of tables and/or plots and outputs only a few significant digits. An option allowing the user to specify that these quantities be printed to a "dump" file from which they can be retrieved by other programs would make it easier to develop and use programs that analyze the results obtained by PEALS.

The information in this section indicates that PEALS is a good software package for finding a few of the eigenvalues of large power systems. Furthermore, it appears that the Arnoldi algorithm is superior to the AESOPS algorithms for most problems. It would be useful if PEALS were modified to output to a "dump" file and to automatically search a user-specified region of the complex $\mathrm{p}$ lane for eigenvalues.

\subsection{CONCLUSIONS}

The programs in the SSSP package are good programs for performing an eigenanalysis of a power system. MASS is best for computing all of the eigenvalues, eigenvectors, and other features of power systems with up to 500 to 1000 states, at least on machines comparable to a VAX 8650 . PEALS, on the other hand, is best suited to computing a few of the features of very large power systems. For most problems, the Arnoldi algorithm in PEALS appears to be superior to the AESOPS algorithm. Methods for computing the eigenvalues of 1 arge, nonsymmetric matrices, however, is an area of active research; as better algorithms are developed, they should be incorporated into the SSSP package. Finally, the possibility of 111 -conditioned eigenvalues suggests 
that it might be useful to modify PEALS and MASS to compute error bounds for some or all of the computed eigenvaiues. A method for computing error bounds is given in Kahan, Parlett, and Jiang (1982). 



\subsection{MASS ON A CRAY SUPERCOMPUTER}

Central processing unit (CPU) time and memory constraints usually limit the use of MASS on conventional computers to probiems with 400 to 500 states (Rogers 1987; Van Ness and Boratynska-Stadnicka 1990). Actual models of power systems, however, are at least an order of magnitude larger than this. Thus, large power system models must be simplified to be analyzed using MASS on a conventional computer, or must be analyzed using other methods.

MASS was modified to run on a Cray supercomputer to examine the feasibility of doing full-scale eigenanalysis of large power systems without having to use simplified models to reduce the size of the problem. The use of MASS on a Cray is described in this section.

\subsection{VECTOR SUPERCOMPUTERS}

The Cray is a vector supercomputer. An excellent description of this class of computers can be found in Levesque and Williamson (1989), while a brief description is provided below.

Every floating point operation on a computer (e.g., addition, multiplication) usually involves several steps or segments, each step taking one or more computer clock cycles to complete. For example, a floating point addition may require three steps, each step taking one clock cycle. Consider a program containing the following FORTRAN statements:

$$
\begin{gathered}
\text { DO } 10 \mathrm{I}=1,1000 \\
A(I)=B(I)+C(I) \\
10 \text { CONTINUE }
\end{gathered}
$$

A conventional computer wil1 compute $B(1)+C(1)$, executing all three steps of the addition, before starting to compute $B(2)+C(2)$ and so on. Thus, it wi11 produce one result every three clock cycles. This mode of operation is called the scaler mode. A vector computer, however, can do the additions in assembly-line (or pipeline) fashion, as illustrated in figure 5.1. This mode of operation is called the vector mode and will produce one result every clock cycle, once the pipeline is filled. Thus, a functional unit with three 


\begin{tabular}{|c|c|c|c|}
\hline Segment 1 & $B(1)+C(1)$ & $B(2)+C(2)$ & $B(3)+C(3)$ \\
\hline Segment 2 & & $B(1)+C(1)$ & $B(2)+C(2)$ \\
\hline Segment 3 & & & $B(1)+C(1)$ \\
\hline
\end{tabular}

FIGURE 5.1. Segmented Adder Operation

segments can produce results three times faster in vector mode than in scaler mode. Actual computers typically use functional units with more than three segments, and vector mode is much faster than scaler mode. Vector mode is used for operations involving vectors, i.e., sections of memory defined by a starting address, a length, and a stride (constant distance between elements in memory). Thus, FORTRAN arrays are vectors, provided that the array elements actually used in a computation are a constant distance apart in memory.

A Cray supercomputer is faster than conventional computers because its CPU is faster in scaler mode than most conventional computers and because of vectorization. Furthermore, vector computers are fairly easy to use because they have compilers that automatically vectorize the innermost loops in computer codes. These compilers also generate ciagnostics describing which loops were vectorized, which were not vectorized, and why they were not vectorized.

Amdahl's Law states that the speedup, i.e., the execution time to run a code in scaler mode divided by the execution time to run the vectorized code, is given by

$$
S=\frac{1}{(1-f)+f / p}
$$

where $S$ is the speedup, $f$ is the fraction of utilized code, i.e., CPUintensive code, vectorized, and $p$ is the speed increase of vector operations over scaler operations (Ortega 1988, p. 29). Let $p=10$. In this case, a computer code will achieve a speedup of 10 if $100 \%$ of the utilized code is 
vectorized, but a speedup of only 2 when $55 \%$ of the utilized code is vectorized. In fact, $90 \%$ or more of the utilized code needs to be vectorized to get a speedup that approaches the maximum possible value. Fortunately, large computer codes typically have only a few CPU-intensive subroutines that need to be optimized to make the code run efficiently on a vector computer.

Two important ways of making the CPU-intensive sections of a computer code run faster on a vector machine are 1) modifying loops that the compiler could not vectorize in such a way that they are now vectorizable (see Levesque and Williamson [1989] for examples of this) and 2) using different algorithms. For example, given a matrix, $A$, there are several algorithms for computing the factorization $A=L U$, where $L$ is a lower triangular matrix and $U$ is an upper triangular matrix; each algorithm does the same arithmetic operations, but in a different order. Each of these algorithms uses different basic vector operations and varies in its efficiency when run on a vector computer (Ortega 1988, pp. 59-65). Consequently, switching to a different algorithm can significantly increase the efficiency of a computer code on a vector computer.

Finally, the Cray does not use virtual memory; thus, the size of problem that can be run is limited by the physical memory available as well as CPU time. Furthermore, the Cray uses a 64-bit word, so the use of single precision on a Cray is equivalent to the use of double precision on 32-bit machines such as a VAX.

\subsection{MODIFICATION OF MASS}

The 4000-bus VAX version of MASS from the Small Signal Stability Program (SSSP) package version 2.0 was converted to run on a cray. This section outlines the major changes that had to be made to run MASS on a Cray. These changes are described in greater detail in Appendix A.

The Cray FORTRAN 77 compiler CFT77 was used for this work. This compiler compites FORTRAN code that conforms to the ANSI FORTRAN 77 standard. It also supports some extensions to this standard, but not all of the extensions supported by the VAX. 
Only some sections of MASS were converted to run on the Cray. The Cray version of MASS 1) reads load flow data in Power Technologies Inc. (PTI) format only, 2) reads dynamic data in Ontario Hydro (OH) format only, and 3) does not allow the use of change case data.

The major changes to the VAX version of MASS to make it run on a Cray were the following:

- INTEGER $* 2$ and INTEGER*4 statements were changed to INTEGER statements.

- Equivalencing of character variables to numeric variables was e]iminated.

- Mixing character and numeric data in common blocks was eliminated.

- Input/output files attached to logical units 5 and 6 were attached to other logical units.

- Statements of the form "STOP 'Tong character string'" were replaced by the pair of statements "WRITE $\left({ }^{*},{ }^{*}\right)$ 'long character string'" and "STOP".

- Calls to the VAX date utility were replaced by calls to the Cray date utility.

- Calls to VAX timing utilities were deleted.

Eliminating the use of INTEGER*2 variables was the only change to MASS that required a significant amount of work. The INTEGER $\star 2$ statement in the section of FORTRAN code

COMMON / WORK / Jl(300), J2(600)

INTEGER $\star 2$ I $1(600)$, I2(1200)

EQUIVALENCE (I1(1), J1(1)), (I2(1), J2(1))

cannot simply be changed to an INTEGER statement, because this would cause array I1 to overlap array 12. In this situation the dimension of Jl must be increased to 600 before the INTEGER 2 statement can be changed to an INTEGER statement. MASS makes extensive use of statements similar to those in the example above. Thus, it was necessary to be careful when eliminating the use of INTEGER*2 statements in MASS.

MASS a1so stores two INTEGER $\star 2$ variables in one INTEGER variable by using statements like 


\section{INTEGER *2 IARRAY(2)}

INTEGER I

EQUIVALENCE (I, IARRAY(1)).

The effect of the above statements must be simulated when INTEGER* 2 variables cannot be used. A method of doing this is described in Appendix A.

\subsection{VECTORIZATION OF MASS}

This section describes what was done to make sure that the Cray version of MASS runs efficientiy on the Cray. The execution times cited in this section are for the T3500 test case supplied with the SSSP package, which has 467 states, to run on a Cray X-MP/18 using version 3.0 of the FORTRAN 77 compiler CFT77 and version 5.0 of the Unicos operating system.

MASS uses subroutines BALANC, ELMHES, ELTRAN, HQR2, and BALBAK from the EISPACK software package (Smith et a1. 1976) to compute the eigenvalues and right eigenvectors of the state matrix. MASS a]so has a set of subroutines, INVERT and GAUS, which compute the left eigenvectors by computing the inverse of the matrix of right eigenvectors. These seven subroutines, which represent only a small fraction of MASS, were the only ones considered when trying to optimize the performance of MASS on the Cray. This was because the total amount of work done by these subroutines varies with $N^{3}$ where $N$ is the number of states, while the work done by all of the other subroutines in MASS, which are associated mainly with input, output and assembling the state matrix, is likely to increase much more slowly as a function of $\mathrm{N}$. The results presented in Section 5.5 show that ignoring the other subroutines when optimizing MASS was reasonable.

The Cray scientific library contains a version of EISPACK. The version of EISPACK in this library, however, appears to be the one described in Smith et a1. (1976), while MASS uses an updated version. For this reason, we vectorized the EISPACK subroutines in MASS rather than simply using the version in the Cray scientific library, to maintain consistency between the original and Cray versions of MASS.

Table 5.1 Tists the execution times of subroutines BALANC, ELMHES, HQR2, INVERT, SGEFA, and SGEDI when running the T3500 problem using several 
IABLE 5.1. Execution Times (sec.) on a Cray X-MP/18

\begin{tabular}{|c|c|c|c|c|c|c|c|}
\hline & BALANC & ELMHES & HOR2 & INVERT & SGEFA & SGEDI & TOTAL \\
\hline Version 1 & 0.38 & 1.68 & 9.45 & 18.76 & - & - & 41.0 \\
\hline Version 2 & 0.38 & 1.66 & 9.86 & 3.13 & - & - & 25.8 \\
\hline Version 3 & 0.38 & 1.66 & 10.27 & 1.80 & 0.60 & 1.2 & 24.9 \\
\hline Version 4 & 0.094 & 1.66 & 10.01 & 1.82 & 0.62 & 1.2 & 24.3 \\
\hline Version 5 & 0.082 & 1.66 & 9.42 & 1.80 & 0.60 & 1.2 & 23.8 \\
\hline
\end{tabular}

different versions of MASS, which are described below. The execution times for subroutines ELTRAN and BALBAK are not listed because they are negligible. The column labeled INVERT is the total execution time for subroutines 1) INVERT and GAUS in rows 1 and 2 and 2) INVERT, SGEFI, and SGEDI in rows 3 through 5. In rows 3 through 5 , the column labeled INVERT is approximately equal to the sum of the columns labeled SGEFA and SGEDI. The column labeled TOTAL is the total execution time for MASS, not just the subroutines listed in the table.

The first version of MASS run on the Cray was obtained by making the minimum number of modifications needed to allow the original VAX version to run on the Cray. The results from this run of MASS are labeled Version 1 in Table 5.1 and show that most of the time was spent in subroutines ELMHES, HQR2, and INVERT (actua1ly GAUS). Thus, these subroutines were modified, when optimizing the performance of MASS on the Cray.

The second version of MASS run on the cray was obtained by making the changes described below to the first Cray version of MASS. Loop 100 in subroutine ELMHES was replaced by a cal1 to the Cray library function ISAMAX. This is the only loop in ELMHES that was not vectorized in the first Cray version of MASS. Calls to a timing routine were added to HQR2 to determine the time spent in several loops, some of which did not vectorize. In subroutine GAUS, 1) loop 1 was replaced by a caTl to the Cray library function ISAMAX, 2) compiler directives were placed at the start of loops 3 and 32 to indicate that it was safe to vectorize these loops, and 3) the array element $D(I)$ in loop 5 was replaced by a scaler. These were the only four loops in GAUS that did not vectorize or were conditionally vectorized. 
The results of running the second version of MASS are labeled Version 2 in Table 5.1. These results show that the change made to ELMHES had a negligible effect on the execution time of this subroutine, while the modifications to subroutine GAUS significantly increased the performance of this subroutine. The timing calls added to HQR2 showed that the total time spent in loops $80,120,140$, and 160 , which did not vectorize, was onty about 0.35 seconds, which is only $1 / 27$ of the time spent in HQR2 in the first cray version of MASS. Thus, little would be gained by trying to vectorize these loops. The only other loops in HQR2 that did not vectorize are defined by the labels 632 and 783. These loops involve only eight to ten iterations on a Cray and, hence, would not benefit from vectorization. Note that the execution time of HQR2 is greater for Version 2 than for Version 1. This can be attributed to adding timing calls to HQR2 in Version 2.

The third version of MASS run on the Cray was obtained by making the changes described below to the second version. In HQR2, loop 40 was replaced by a call to the Cray library routine SASUM, while 100ps 610, 760, and 880 were replaced by calls to the Cray library routine SDOT. Also, subroutine GAUS was replaced by the LINPACK subroutines SGEFA and SGEDI (Dongarra et al. 1979). Subroutine SGEFA computes the LU factorization of a matrix using essentially the same algorithm used in subroutine GAUS. Subroutine SGEDI computes the inverse of a matrix, which has been factored using SGEFA, using an algorithm that requires $2 / 3 \mathrm{~N}^{3} \mathrm{~T}$ seconds to execute, while GAUS uses an algorithm that requires $N^{3} T$ seconds, where $N$ is the dimension of the matrix and $T$ is the time (seconds) required by a given computer to execute the FORTRAN statement

$$
Y(I)=Y(I)+A \star X(I)
$$

once (Dongarra et al. 1979, p. 1.22). This timing comparison is only approximately correct for vector computers. Furthermore, SGEDI uses more efficient vector operations than the algorithm used in GAUS. Before using SGEFA, 1) calls to the LINPACK routines SAXPY and SSCAL were replaced by vectorizable, intine code, 2) it was modified to set an error flag if the absolute value of any pivot was $<10^{-10}$ (done to be consistent with GAUS; however, it would probably be better to compute the condition number [Dongarra et a]. 
1979, Chapt. 1] of the matrix to be inverted), and 3) row interchanges were modified to be vector operations. Finally, calls to the LINPACK routines SAXPY, SSWAP, and SSCAL were replaced by vectorizable, inline code before using SGEDI.

The results of running the third version of MASS are labeled Version 3 in Table 5.1. These results, and timing data that are not listed, show that replacing the loops in HQR2 by calls to the Cray library routines slightly increased the execution times of these loops. The results, however, show that using the LINPACK routines in $\mathrm{place}$ of GAUS yielded a significant improvement in performance. In fact, replacing GAUS by these LINPACK routines also improves the performance of MASS on conventional computers such as a VAX.

A fourth Cray version of MASS was created in which the Cray scientific library versions of subroutines BALANC, ELMHES, ELTRAN, HQR2, BALBAK, SGEFA, and SGEDI were used in place of the MASS versions. This was to ensure that the MASS versions of these subroutines were at least comparable in speed to the Cray scientific library versions. The results of using this version are labeled Version 4 in Table 5.1. These results show that the vectorized MASS versions of all these subroutines, except BALANC, are as fast as, or slightly faster than, the Cray library versions. The execution times for this version should be compared to the results labeled Version 3, except that the results for HQR2 should be compared to the results labeled Version 1. This suggests that these subroutines have been vectorized fairly well.

A final Cray version of MASS, CMASS, was created. It consisted of subroutines 1) ELMHES, INVERT, SGEFA, and SGEDI from Version 3 above, 2) HQR2 from Version 1 above, and 3) a modified version of BALANC (10op 200, which does not vectorize, was replaced by two loops, which do vectorize) from Version 1 above. The results of using CMASS are labeled Version 5 in Table 5.1. Note that the new version of BALANC is slightly faster than the version in the Cray library.

Table 5.2 Tists speedup data for CMASS. The first row gives the execution times for CMASS when compiled normally (this is the same as the row labeled Version 5 in Table 5.1), while the second row gives the execution times for CMASS when compiled with vectorization turned off. The last row 
TABLE 5.2. Speedup on a Cray X-MP/18

Execution time (sec)

$\begin{array}{llcrrrrr} & \text { BALANC } & \text { ELMHES } & \text { HOR2 } & \text { INVERT } & \text { SGEFA } & \text { SGEDI } & \text { TOTAL } \\ \text { Vector mode } & 0.082 & 1.65 & 9.43 & 1.80 & 0.60 & 1.2 & 23.8 \\ \text { Scaler mode } & 0.26 & 23.6 & 72.86 & 2 B .25 & 9.3 & 18.9 & 143.9\end{array}$

Speedup

$3.2 \quad 14.3$

$\begin{array}{ll}7.7 & 15.7\end{array}$

15.5

15.8

6.0

gives the speedup, i.e., the time to execute without vectorization divided by the time to execute with vectorization. The results in Table 5.2 show that subroutines ELMHES, INVERT, SGEFA, and SGEDI are very we11 vectorized. Subroutine BALANC is only slightly vectorized, but this is acceptable because its total execution time is negligible. Finally, subroutine HQR2 is reasonably well vectorized.

Additional improvements in the performance of HQR2 can be achieved (Dongarra, Kaufman, and Hammarling 1986), but require significantly more code modification than was described above. Furthermore, a new software package, LAPACK (Bischof and Dongarra 1989), is scheduled for release in early 1991. This package combines LINPACK and EISPACK into a single package and uses algorithms that are designed to run more efficiently on vector computers, such as the Cray, than the algorithms in LINPACK and EISPACK. Thus, the most efficient way to improve the performance of CMASS on the Cray would be to wait for the release of LAPACK and then to replace the LINPACK and EISPACK routines in CMASS with LAPACK routines.

The total execution time of the subroutines listed in Table 5.2 is only 13 seconds (the INVERT column includes the times for subroutines SGEFA and SGEDI), while the total execution time of MASS was 24 seconds. Thus, it may appear that vectorization of other sections of CMASS could significantly improve the performance of CMASS. As noted at the beginning of this section, however, the eigenvalue computations should dominate all of the other computations for large problems, in which case vectorizing other sections of CMASS would provide little benefit. The results presented in Section 5.5 verify that this assumption is correct. 
The final Cray version of MASS, dimensioned the same as the original 4,000-bus, VAX version of MASS, requires $1,848,885$ words of memory and a 11 ows

up to 500 states. In general, CMASS requires approximately $4 N^{2}+1,202,000$ words of memory, where $\mathrm{N}$ is the number of states for which CMASS is dimensioned. This formula is based on the memory requirements for a 2200-state version of CMASS and overestimates the memory required when $N$ is small or moderately large.

\subsection{IESTING OF CMASS}

The data supplied by the Electric Power Research Institute (EPRI) with the SSSP package includes six test problems for use with MASS. The Cray version of MASS was tested to verify that the results produced by the EPRI-supplied VAX version of MASS and the new Cray version of MASS produce similar results on these problems. Appendix $B$ describes the testing that was done to ensure that the Cray version of MASS, CMASS, works correctly. This section summarizes the results presented in Appendix B.

While testing was in progress, it was found that CMASS uses uninitialized variables and assumes that these variables have a value of zero. Thus, CMASS must be compiled with the "-a static" option on the Cray X-MP EA/232. Otherwise, uninitialized variables may have nonzero values and CMASS may not work correctly on some problems. Furthermore, it was found that the matrix of right eigenvectors for the $T 3500$ problem is $i 11$-conditioned. This caused some of the left eigenvectors computed for the $T 3500$ problem, by the original VAX version of MASS, to have only one or two correct decimal digits. This shows the importance of modifying MASS to compute and print the condition number of the matrix of right eigenvectors, as was recommended in Section 4 . Furthermore, this suggests that it would be a good idea for the VAX version of MASS to use double precision when computing the matrix of left eigenvectors, at least when the matrix of right eigenvectors is $i 11$-conditioned.

The results in Appendix $B$ show that the differences between the results computed by the Cray and VAX versions of MASS can reasonably be attributed to the fact that the Cray uses 64-bit arithmetic while the VAX uses 32-bit arithmetic, with one possible exception. A few of the entries in the $T 3500$ 
state matrices computed by the Cray and VAX versions of MASS are moderately different. It appears reasonable that these differences are the result of the Cray using 64-bit arithmetic and the vaX using 32-bit arithmetic, but they may be the result of an error in the Cray version of MASS. The actual cause of these differences has not been found because of time limitations. However, it should determined before the cray version of MASS is used for power system analysis.

Overa11, the tests conducted so far give a reasonably good indication that the Cray version of MASS works correctiy. The test problems run, however, do not exercise all of the options available in MASS. Consequently, it is recommended that the Cray version of MASS undergo additional testing before it is used for analyzing power systems.

\subsection{PERFORMANCE OF MASS ON A CRAY}

This section compares the performance of MASS on a Cray X-MP EA/232 and on conventional computers.

The Cray X-MP/18 (one central processor, 8 megawords of memory) initially used for this project was replaced by a Cray X-MP EA/232 (Extended Architecture, two central processors, 32 megawords of memory) shortly after the work just described was completed. Extended architecture means 32-bit address capability as opposed to the Cray X-MP/18's 24-bit address. The largest job that can nomally be run on this Cray X-MP EA/232 (using the night or weekend queue) is 8 megawords. With special permission, however, it is possible to run jobs as large as 16 megawords on these queues. A11 of the Cray results in this section were obtained using the FORTRAN 77 compiler CFT77 version 3.1.2.6 on the Cray X-MP EA/232.

Three test problems were run on the cray.

The T3500 test case is supplied by EPRI with the SSSP package. It has 3554 buses and 467 states. There are 537 generators in the powerflow, but only 53 generators are used in the dynamic data file. This problem models the 
power system in Florida, load level and month unknown, but the bus names have been changed because some utilities consider their network data to be proprietary.

The BCH88HW3 problem is a January 1988 peak load Western Systems Coordinating Council (WSCC) base case for use with PEALS (one of the programs in the SSSP package). It has 2304 buses and 374 generators in the powerflow; all of the generators are included in the dynamic data file. This problem was used by the WSCC 0.7 Hertz Osci11ation Ad Hoc Work Group. PEALS indicates that the BCH88HW3 test case has 3873 states. If CMASS were dimensioned large enough to run this problem, it would require approximately 60 megawords of memory, which greatly exceeds the 16 -megaword job limit. Thus, two new test cases, BCH88HW3_2 and BCH88HW3_3, were created by modifying the original BCH88HW3 case. The $\mathrm{BCH}_{88 \mathrm{HW}}{ }_{2} 2$ test case was obtained by deleting the controls from all of the machines, except for those located in the Northwest (i.e., at buses 718 through 1298), in the BCH88HW3 dynamic data file. The BCH88HW3_3 test case was obtained by modifying the classical generator data in the BCH88HW3_2 dynamic data file so that MASS will model the classical machines as classical machines (which is what PEALS always does) rather than as infinite buses. It was found, using PEALS, that both the BCH88HW3_2 and the BCH88HW3_3 test problems have 2165 states (the $\mathrm{BCH} 88 \mathrm{HW} 32$ problen will have less than 2165 states when run using MASS because MASS will model the classical machines as infinite buses), which exceeds the biggest problem that CMASS can run on the Cray. A modified version of CMASS, CMASS_2200, was used to solve the $\mathrm{BCH} 88 \mathrm{HW} 32$ and $\mathrm{BCH}_{3} 8 \mathrm{HHW}_{3}{ }_{3}$ problems on the Cray.

The differences between CMASS and CMASS_2200 are that 1) the subroutines that do frequency response calculations are not oresent in CMASS 2200, 2) the arrays in CMASS_2200 are dimensioned 1arger than in CMASS, and 3) CMASS_2200 prints out only a limited number of eigenvectors and participation vectors. A detajled description of these differences is given in Appendix $C$. Deleting the frequency response subroutines from CMASS reduced the memory requirements from about $4 \mathrm{~N}^{2}+1,202,000$ words of memory to about $3 \mathrm{~N}^{2}+1,202,000$ words of memory. This allowed CMASS_2200 to be dimensioned large enough for the BCH88HW3_3 problem and sti11 run on the Cray. Limiting the amount of output 
done by CMASS_2200 was done so that some participation vectors and eigenvectors could be printed without having an excessively large output file.

Table 5.3 Tists the execution times of the T3500, BCH88HW3_2, and BCH88HW3 3 test problems on several different machines. The T3500 problem has 467 states, the BCH88HW3_2 problem has 1997 states, and the BCH88HW3_3 problem has 2165 states. The Cray times are 1) the time required to run the $T 3500$ problem using CMASS and 2) the times to run the BCH88HW3_2 and BCH88HW3_3 problems using CMASS_2200 (running the T3500 problem using CMASS_2200 gave essentially the same times as using CMASS). The VAX times were obtained using the original 4000-bus, VAX version of MASS.

Subroutines ELMHES, HQR2, SGEFA, and SGEDI in CMASS_2200 used approximateTy 95.6 percent (96.8 percent) of the CPU time when running the BCH88HW3_2 (BCH88HW3_3) test problem on the Cray. This shows that optimizing any other subroutines in CMASS would yield a negligible decrease in execution times on the cray when running large problems. This is the result predicted in Section 5.3.

Generally, the execution time of the main computational subroutines ELMHES, HQR2, SGEFA, and SGEDI in CMASS will be proportional to $N^{3}$, where $N$ is the number of states in the problem. Thus, the execution time of CMASS should be proportional to $\mathrm{N}^{3}$, provided that the problem is large enough that CMASS spends most of its time in these subroutines. Using this $\mathrm{N}^{3}$ rule, the execution time of the BCH88HW3 3 problem should be approximately 1.274 times as long as the execution time of the BCH8BHW3_2 problem. This yields a predicted execution time of about 1566 seconds for the BCHB8HW3_3 problem, which is essentially the same as the actual execution time of 1568 seconds for this problem. Furthermore, the execution time of the BCH88HW3_2 problem is about

TABLE 5.3. MASS Execution Times on Various Computers

\begin{tabular}{|c|c|c|c|}
\hline & \multicolumn{3}{|c|}{ Execution Time (seconds) } \\
\hline & $\overline{T 3500}$ & BCH88HW3 2 & $\mathrm{BCH88HW3} 3$ \\
\hline Cray X-MP EA/232 & 26 & 1229 & 1568 \\
\hline VAX 8650 & 2172 & - & - \\
\hline MicrovaX & 6708 & - & - \\
\hline
\end{tabular}


what one would expect based on the $N^{3}$ rule and the execution times of subroutines ELMHES, HQR2, SGEFA, and SGEDI in the T3500 case (data not shown). These results show that the execution time of CMASS on the Cray X-MP EA/232 will be approximately $2.575 \times 10^{-9} \times N^{3}$ minutes, where $N$ is the number of states, provided that $N$ is greater than about 1000 . Finally, the $N^{3}$ rule implies that the $\mathrm{BCH} 88 \mathrm{HW} 33$ problem would require approximately 2.5 days of CPU time on the VAX 8650 , while page fautts would probably cause the actual execution time to be significantly longer than this.

The cost of running a job on the Cray is determined mainly by the amount of CPU time used and which queue is used. The estimated cost of running the BCH88HW3 3 test problem on the Cray is $\$ 63$ on the night queue or $\$ 32$ on the weekend queue. The estimated cost of running the T3500 problem on the cray is $\$ 2.30$ on the day queue or $\$ 1.16$ on the night queue.

\subsection{CONCLUSIONS}

The results of this section have shown that a Cray version of MASS can easity compute all of the eigenvalues and eigenvectors of power systems with up to 1900 states, though this can be increased to 2200 states by deleting the frequency response subroutines from MASS. Many power system problems, however, are significantly larger than these limits.

The factor limiting the size of problem that could be solved during this project was the 16-megaword job size limit on the Cray used for the project. There are several ways of increasing the size of problem that can be solved by a Cray version of MASS. The easiest method is to run MASS on a larger Cray. The next easiest would be to reduce the memory requirement of MASS from $4 N^{2}+1,202,000$ words to $2 N^{2}+1,202,000$ words. The most difficult method would be to develop an out-of-core version of MASS.

There exist Crays with up to 512 megawords of memory, though there are only a few of the largest machines around. Problems with as many as 8,000 to II,000 states could be solved by running MASS on a 512-megaword machine, depending on whether a $4 N^{2}+1,202,000$-word or a $2 N^{2}+1,202,000$-word version of MASS is used, assuming that any given job can use approximately half of the machine's memory. It would take approximately 22 to 57 Cray hours to solve 
problems with 8,000 to 11,000 states, based on the execution time of the $\mathrm{BCH} 8 \mathrm{BH}$ 3_3 test problem and the $\mathrm{N}^{3}$ execution time rule. A 5000-state problem, however, would require only about 5.3 Cray hours. Some Crays are faster than others, however, so the actual execution times would depend upon the type of cray used.

A problem requiring about 5 Cray hours is very reasonable, and would probably cost less than a thousand dollars, though the actual cost depends on the billing algorithm on the cray being used. Problems requiring 22 to 57 Cray hours can be done, but will probably cost several thousand dollars. Furthermore, a $2 \mathrm{~N}^{2}+1,202,000$-word version of MASS would require only about 52 megawords of memory to solve a 5000-state problem. Thus, a Cray with 128 or maybe just 64 megawords of memory could be used to solve these problems. This is useful because the smatler Crays are more readity available than Crays with 512 megawords of memory. Thus, the practical limit for the size of problem that could be routinely solved using MASS on a cray is probably about 5000 states, though problems with 10,000 or so states could be solved if necessary.

Developing a $3 N^{2}+1,202,000$-word version of MASS would probably be fairly easy, while developing a $2 \mathrm{~N}^{2}+1,202,000$-word version would probably be more difficult, as noted in Section 4. Whether developing these reduced memory versions of MASS is worthwhile depends on 1) the size of Cray available for use with MASS and 2) whether the increase in the size of problem that could be solved by MASS on the available Cray is considered to be worth the effort required to modify MASS.

Solid-state disks (SSDs) are available for use with Crays. SSDs are a very fast form of secondary memory and make out-of-core computations feasible. Creating an out-of-core version of MASS would increase the size of problem that could be solved by any given Cray, but 1) it would require a substantial amount of work, and 2) it would be slower than an in-core version of MASS. Furthermore, if a large cray is used, then the factor limiting the size of problem that can be solved is CPU time, not memory. Thus, developing an outof-core version of MASS is probably not worthwhile. 
Most of the larger Crays have several processors. Consequent7y, it is possible to do computations in paralle1. Furthermore, the Cray has an autotasking compiler that will automatically parallelize FORTRAN code to a certain extent. If autotasking produces good results on MASS, then the actual turnaround time for solving large problems may be significantly less than the total CPU time required by the problem, though the cost of the job would probably not be reduced. Whether autotasking would work well on MASS is unknown, but it may be worth investigating if MASS is used to solve problems with more than about 5000 states.

In conclusion, it appears that it should be possible to use a Cray version of MASS to routinely solve power system problems with up to about 5000 states, though problems with about 10,000 states could probably be solved if necessary. As Crays become larger and faster, however, these limits will increase. The only requirements for increasing the problem 1 imit from the current value of 1,900 to 2,200 states to 5,000 to 10,000 states are to 1) move to a larger Cray, and 2) modify MASS to use only $2 \mathrm{~N}^{2}+1,202,000$ or $3 N^{2}+1,202,000$ words of memory. The second modification may not be necessary if MASS is moved to a large enough cray. Finally, the types of output done by MASS should probably be modified to reduce the size of output files. 


\subsection{GRAPHICS AND EIGENANALYSIS}

When studying the small signal stability of a power system, one is typically interested in the answers to two questions:

- Do any of the eigenvalues of the power system lie in a specific region of the complex plane?

- Which parts of the power system contribute significantly to the modes whose eigenvalues lie in the specified region of the complex p]ane?

Usually the region of interest will be the right half of the complex plane (which corresponds to unstable modes) and/or the region near the imaginary axis (which corresponds to poorly damped modes).

To help answer these questions, some simple graphical methods for portraying the results produced by a typical power system eigenanalysis package are considered in this section. The various quantities produced by a typical eigenanalysis program were described in Section 2 and include eigenvalues, right and left eigenvectors, and participation vectors.

The easiest way to visualize the locations of the eigenvalues of a power system is to plot them in the complex plane. This is a fairly common technique. Coloring or outlining the specific region of interest would allow the eigenvalues of interest to be distinguished from the remaining eigenvalues. Alternatively, one could plot only the eigenvalues lying within the region of interest. The eigenvalues of nonsymmetric matrices, however, may be illconditioned, in which case roundoff errors may cause moderately large errors in the computed values of some of the eigenvalues. Thus, one may be interested in whether some of the eigenvalues that were computed to be outside of the region of interest may actually 7 ie within the region of interest.

It is possible, using the right and left eigenvectors, to obtain error bounds for the computed eigenvalues (Kahan, Parlett, and Jiang 1982). Thus, one may want to compute error bounds for the eigenvalues near the region of interest and highlight any eigenvalues whose true value may be within the region of interest. Computing error bounds for the eigenvalues will probably have to be done by the actual eigenanalysis code. Consequently, it would be 
useful if the eigenanalysis code had an option that allowed the user to specify a region of the complex plane such that error bounds will be computed for all eigenvalues in the specified region.

The types of plots just described allow one to visually examine the eigenvalue distribution of a power system. They are much easier to interpret than a printout listing the eigenvalues.

Given an eigenvalue of interest, one usually wants to know which parts of the power system participate in that mode, and how strongly. The right eigenvector of the corresponding eigenvalue gives the relevant information. In particular, the relative phases and magnitudes of the various state variables are determined by the relative phases and magnitudes of the components of the eigenvector. One way to visualize the information in the right eigenvector is to plot the components of the vector on a geographical map.

To illustrate this method, let $\lambda_{j}$ be the eigenvalue of interest and let $w_{i, k}$ be the kth component of the corresponding right eigenvector. $w_{i, k}$ can then be plotted as a vector such that 1) the tail of the vector is located on the part of the map corresponding to the physical location of the kth state variable; 2) the horizontal component of the vector is equal to the real part of $w_{i, k}$; and 3 ) the vertical component of the vector is equal to the imaginary part of $w_{i, k}$. In practice, one may be interested only in certain types of state variables, e.g., the state variables corresponding to generator angle or generator speed. Thus, it would be useful to be able to plot only those components of the right eigenvector that correspond to certain types of state variables.

Figures 6.1 and 6.2 are examples of these plots. Both plot the right eigenvector associated with the first eigenvalue computed by the Cray version of MASS for the BCH88HW3_3 test problem (see Section 5). In Figure 6.1, those components of the right eigenvector that correspond to generator speed are plotted; Figure 6.2 plots those components of the eigenvector that correspond to generator angle. These plots provide a simple way of determining which 


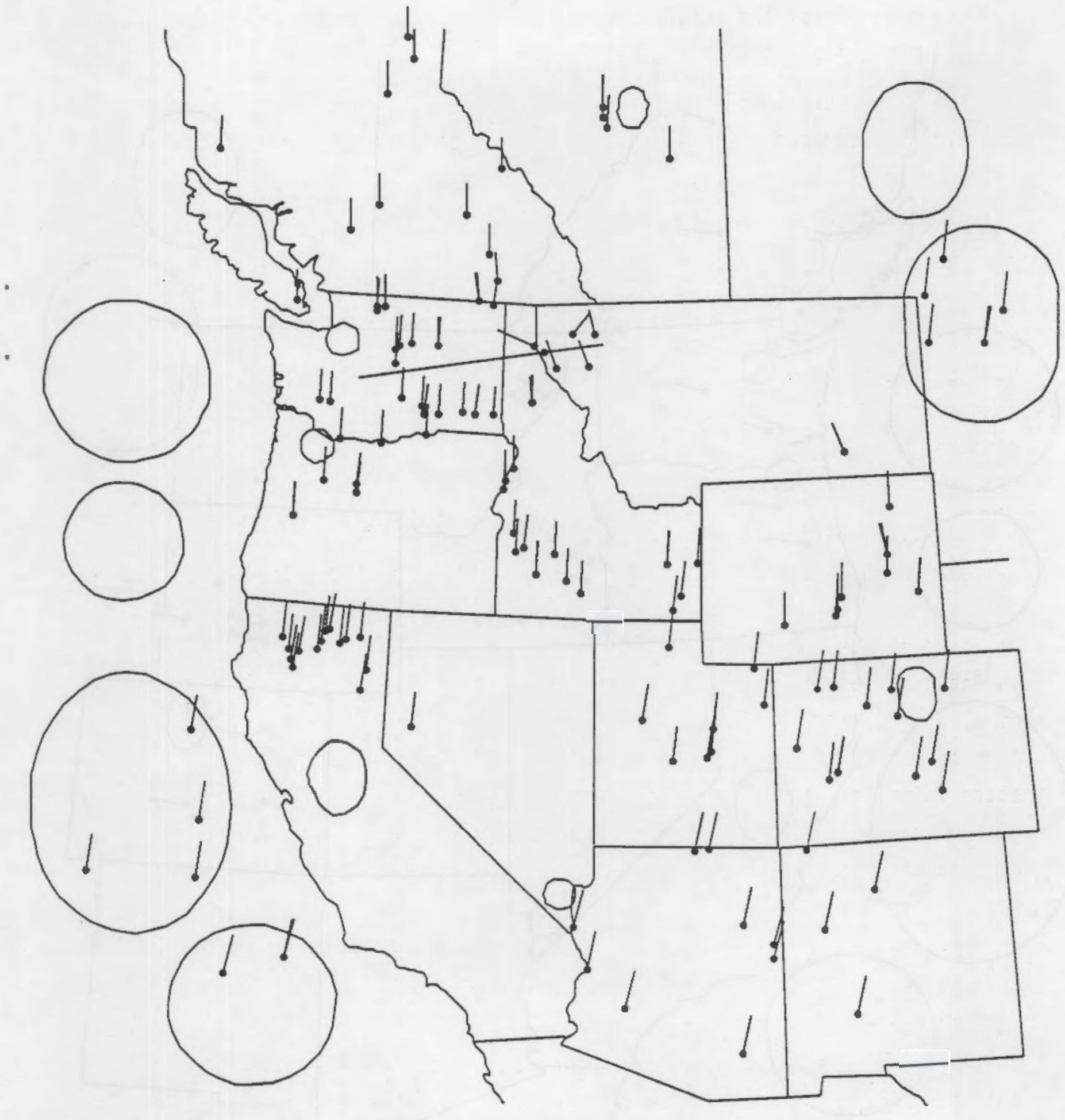

FIGURE 6.1. Generator Speed 


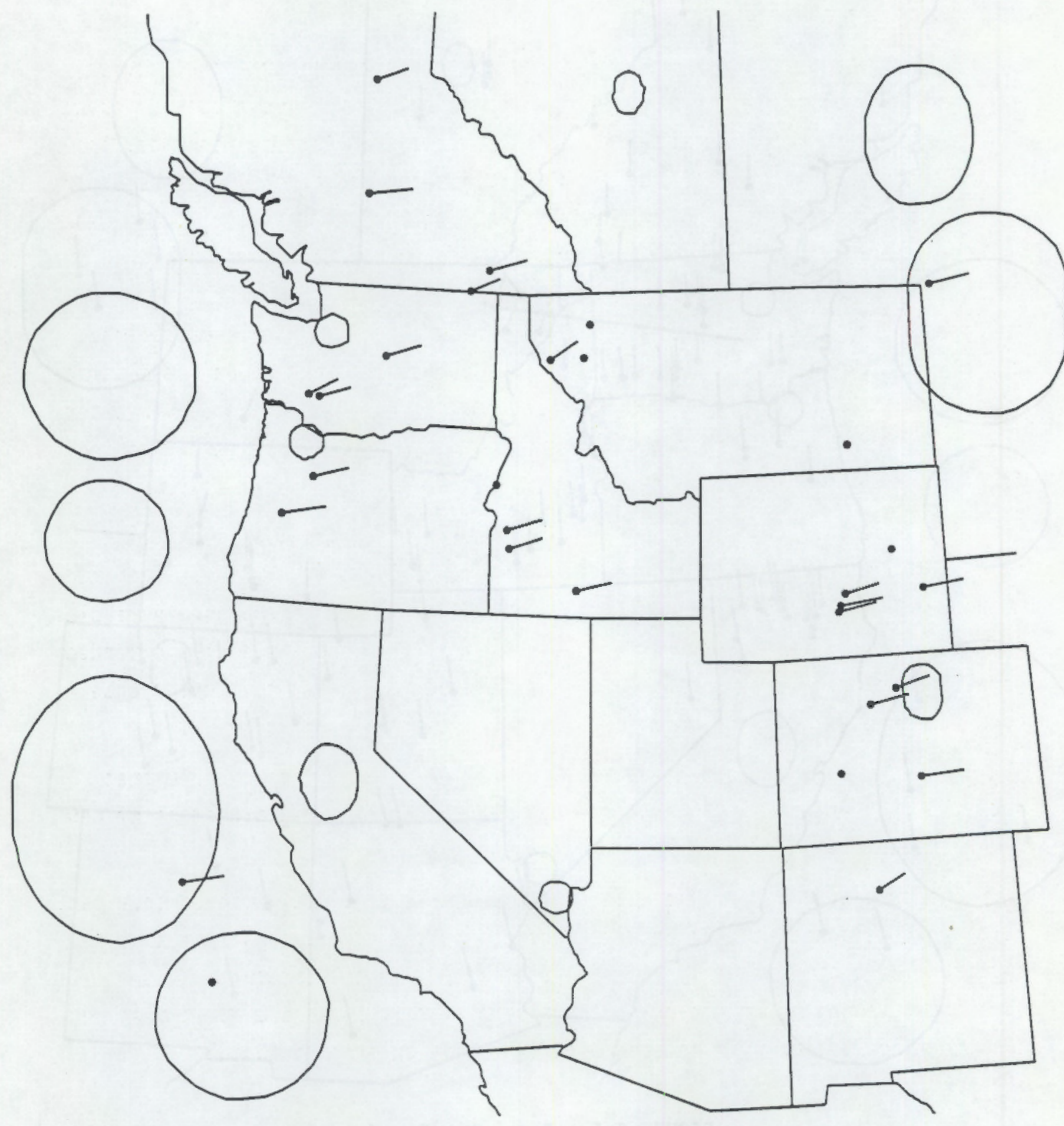

FIGURE 6.2. Generator Angle 
part of the power system participates significantly in a given mode. These plots also allow the relative phase and magnitude of the mode to be visualized throughout the power system.

The relative magnitudes of the various components of a right eigenvector are dependent on the unit of measurement used for the various state variables. The participation vectors are dimensioniess, however. As a consequence, the relative magnitudes of their components are independent of the units used for the state variables, providing a natural scaling for the relative magnitudes of the various state variables in a mode. An alternative type of plot would be to rescale the right eigenvector plots described above so that the magnitudes of the various vectors are proportional to the magnitudes of the corresponding participation factors. The phase of the vectors in the alternative plot would then be determined by the components of the right eigenvector, while the magnitudes of the vectors would be determined by the participation factors.

The eigenvector plots described above could be enhanced if the method allowed the user to select a specific state variable as a reference, and then use one color for all variables that are in phase with the reference variable and different colors for the other variables. This feature could be extended to allow the user to select several reference variables and then color code each variable based on which reference variable it is in phase with.

Finally, we note that it would be useful if the graphics program that does the eigenvector plots were to allow for the interactive placement of new power system locations on the digitized map.

The preceding paragraphs describe some simple graphical methods that can be used to help analyze the results produced by the eigenanalysis of a power system. There are certainly other ways of analyzing these results, but developing the necessary graphics and expert systems for these would best be done in consultation with someone with extensive experience in the eigenanalysis of power systems. 


\subsection{CONCLUSIONS}

The eigenanalysis of power systems with up to 500 states is routinely done on conventional computers using programs based on the $Q R$ algorithm. Large power systems, however, are characterized by many more states, so simplified models must be used if the QR algorithm is to be applied effectively to their analysis. These simplified models are not as accurate as the detailed models; thus, other methods that can perform the analyses without resorting to the simplified models are needed. Development of CMASS, a Cray version of MASS, indicates that the use of the QR algorithm can be extended to problems with 5,000 to 10,000 states. In addition, a number of new methods have been developed in recent years to do the eigenanalysis of large power systems.

Development of CMASS has shown that problems with about 2200 states can be solved on a Cray X-MP EA/232 in about 26 minutes. By moving to larger machines, however, it should be possible to solve problems with 5,000 to 10,000 states. The 5,000 -state problems would probably take about 5.3 Cray hours, while a 10,000-state problem would probably take about 43 Cray hours, though these times depend on the actual machine used. A job requiring 5.3 Cray hours is not unreasonable and would probably not be too expensive. A job requiring 43 Cray hours, however, is very large and would be fairly expensive. Thus, the practical upper limit for the size of problem that could be routinely solved using MASS on a Cray is probably around 5000 states. As Crays become larger and faster, however, this ceiling will rise.

For power systems with more than a few thousand states, one must turn to eigenanalysis methods that do not use the QR algorithm. The general algorithms for finding some of the eigenvalues of large, sparse, nonsymmetric matrices, such as simultaneous iteration, variations of Arnoldi's method, and variations of the Lanczos method, appear to be the best options for the eigenanalysis of these systems. However, the other algorithms described in Section 3 may be better for some problems. Those algorithms appear to work fairly wel1. Nevertheless, the computation of eigenvalues of large, sparse, nonsymmetric matrices is still an active research area. New developments in 
this area should be watched closely, and new algorithms should be incorporated into power system eigenanalys is packages when appropriate.

The eigenanalysis of large power systems produces considerable information. Some simple graphics methods described in Section 6 can be used to help analyze this information. It would be useful, however, if more advanced methods could be developed for assisting power system planners and analysts in using this information to determine the major sources of instability in a power system. Such advanced methods should be developed with the assistance of people with extensive experience in power systems eigenanalysis. 


\subsection{REFERENCES}

Anderson, P. M., B. L. Agrawal, and J. E. Van Ness. 1990. Subsynchronous Resonance in Power Systems. IEEE Press, New York.

Bischof, C. H., and J .J. Dongarra. 1989. "A Linear Algebra Library for High-Performance Computers." Chapter 4 in Parallel Supercomputing: Methods, Algorithms and Applications, ed. G. F. Carey, pp. 45-56. John Wiley \& Sons, New York.

Boley, D., R. Maier, and J. Kim. 1989. "A Parallel QR Algorithm for the Nonsymmetric Eigenvalue Prob7em." Comp. Phys. Comm, 53:61-70.

Byerly, R. T., R. J. Bennon, and D. E. Sherman. 1982. "Eigenvalue Analys is of Synchronizing Power Flow Oscillations in Large Electric Power Systems." IEEE Trans. Pow. App. Sys, 100:235-243.

Cullum, J. K., W. Kerner, and R. A. Willoughby. 1989. "A Generalized Nonsynmetric Lanczos Procedure." Comp. Phys. Comm. 53:19-48.

Cullum, J. K., and R. A. Willoughby, 1984. "A Lanczos Procedure for the Modal Analys is of Very Large Nonsymmetric Matrices." In Proceedings of the 23rd Conference on Decision and Control, pp. 1758-1761. December 1984, Las Vegas, Nevada. Institute of Electrical and Electronics Engineers, New York.

Cullum, J. K., and R. A. Willoughby. 1985. Lanczos Algorithms for Large Symetric Eigenvalue Computations, Vol. I Theory. Birkhauser, Boston.

Dandeno, P. L., and P. Kundur. 1976. "Practical Application of Eigenvalue Techniques in the Analysis of Power System Dynamic Stability Problems." Can. Elec. Eng. J. 1:35-46.

Dongarra, J. J., L. Kaufman, and S. Hammarling. 1986. "Squeezing the Most out of Eigenvalue Solvers on High-Performance Computers." Lin. Alg. Appl. $77: 113-136$.

Dongarra, J. J., C. B. Moler, J. R. Bunch, and G. W. Stewart. 1979. LINPACK User's Guide. SIAM, Philadelphia.

Geist, G. A., and G. J. Davis. 1990. "Finding Eigenvalues and Eigenvectors of Unsymmetric Matrices Using a Distributed-Memory Multiprocessor." Paral. Comp. 13:199-209.

Golub, G. H., and C. F. Van Loan. 1983. Matrix Computations. John Hopkins University Press, Baltimore, Maryland.

Gross, G., C. F. Imparato, and P. M. Look. 1982. "A Tool for the Comprehensive Analysis of Power System Dynamic Stability." IEEE Trans. Pow. App. Sys. 101:226-234. 
Hauer, J. F. 1990. "Introduction to the 1989 1EEE/PES Symposium on Eigenanalysis and Frequency Domain Methods for System Dynamic Performance." In Eigenanalysis and Frequency Domain Methods for System Dynamic Performance, pp. 1-4. IEEE publication 90TH0292-3-PWR, Institute of Electrical and Electronics Engineers, New York.

Ho, D. 1990. "Tchebychev Acceleration Technique for Large Scale Nonsymnetric Matrices." Numer. Math. 56:721-734.

Jennings, A., and W. J. Stewart. 1975. "Simultaneous Iteration for Partial Eigensolution of Real Matrices." J. Inst. Math. Applics. 15:351-361.

Kahan, W., B. N. Parlett, and E. Jiang. 1982. "Residual Bounds on Approximate Eigensystems of Nonnormal Matrices." SIAM J. Num. Anal. 19:470-484.

Kerner, W. 1989. "Large-Scale Complex Eigenvalue Problems." J. Comp. Phys. $85: 1-85$.

Kundur, P., G. J. Rogers, and D. Y. Wong. 1988. The Small Signal Stability Program Package. Volume 1: Program Package Development. EPRI EL-5798, Electric Power Research Institute, Palo Alto, California.

Levesque, J. M., and J. M. Witliamson. 1989. A Guidebook to Fortran on Supercomputers. Academic Press Inc., New York.

Martins, N. 1986. "Efficient Eigenvalue and Frequency Response Methods Applied to Power System Small-Signal Stability Studies." IEEE Trans. Pow. Sys. 1:217-226.

Ortega, J. M. 1988. Introduction to Parallel and Vector Solution of Linear Systems. Plenum Press, New York.

Parlett, B. N. 1980. The Symmetric Eigenvalue Problem. Prentice-HaT1, Englewood Cliffs, N.J.

Parlett, B. N. 1984. "The Software Scene in the Extraction of Eigenvalues from Sparse Matrices." SIAM. J. Sci. Stat. Comput. 5:590-604.

Parlett, B. N., D. R. Taylor, and Z. A. Liu. 1985. "A Look-Ahead Lanczos Algorithm for Unsymmetric Matrices." Math. Comp. 44:105-124.

Perez-Arriaga, I. J., G. C. Verghese, and F. C. Schweppe. 1982. "Selective Moda] Analysis with Applications to Electric Power Systems, Part I: Heuristic Introduction." IEEE Trans. Pow. App. SYS. 101:3117-3215.

Rogers, G. J. 1987. "Methods for Small Signal Analysis of Very Large Power Systems." In Proceedings of the 26th Conference on Decision and Control, pp. 393-398. December 1987, Los Angeles, California. Institute of Electrical and Electronics Engineers, New York. 
Rogers, G. J., and P. Kundur. 1990. "Small Signal Stability of Power Systems." In Eigenanalysis and Frequency Domain Methods for System Dynamic Performance, pp. 5-16. IEEE pub1ication 90TH0292-3-PWR, Institute of Electrical and Electronics Engineers, New York.

Ruhe, A. 1983. "The Two-Sided Arnoldi Algorithm for Nonsymmetric Eigenvalue Problems." In Matrix Pencils: Proceedings of a Conference, eds. B. Kagstrom and A. Ruhe, pp. 104-120. March 22-24, 1982, Pite Havsbad, Sweden. Lecture Notes in Mathematics, Vo1. 973, Springer-Verlag, New York.

Saad, Y. 1980. "Variations on Arnoldi's Method for Computing Eigenelements of Large Unsymmetric Matrices." Lin. Alg. Appl. 34:269-295.

Saad, Y. 1984. "Chebyshev Acceleration Techniques for Solving Nonsymmetric Eigenvalue Problems." Math. Comp. 42:567-588.

Saad, Y. 1989. "Numerical Solution of Large Nonsymmetric Eigenvalue Problems." Comp. Phys. Comm. 53:71-90.

Semlyen, A., and L. Wang. 1988. "Sequential Computation of the Complete Eigensystem for the Study Zone in Small Signal Stability Analysis of Large Power Systens." IEEE Trans. Pow. Sys, 3:715-725.

Smith, B. T., J. M. Boyle, J. J. Dongarra, B. S. Garbow, Y. Ikebe, V. C. Klema, and C. B. Moler. 1976. Matrix Eigensystem Routines - Eisoack Guide. Springer-Verlag, New York.

Sorensen, D. C. 1990. Implicit Applications of Polynomial Filters in a k-Step Arnoldi Method. Technical Report TR90-27, Department of Mathematical Sciences, Rice University, Houston, Texas.

Stewart, W. J., and A. Jennings. 1981. "A Simultaneous Iteration ATgorithm for Rea1 Matrices." ACM Trans. Math. Software 7:184-198.

Uchida, N., and T. Nagao. 1988. "A New Eigen-Analysis Method of SteadyState Stability Studies for Large Power Systems: S Matrix Method." IEEE Trans. Pow. Sys. 3:706-714.

Undri11, J. M. 1968. "Dynamic Stability Calculations for an Arbitrary Number of Interconnected Synchronous Machines." IEEE Trans. Pow. App. Sys. 87:835844 .

Van Ness, J. E., and D. J. Boratynska-Stadnicka. 1990. "A Partitioning Algorithm for Finding Ejgenvalues and Eigenvectors." In Proceedings of the Tenth Power Systems Computation Conference, pp. 91-98. August 19-24, 1990, Graz, Austria. Butterworths, Boston.

Van Ness, J. E., F. M. Brasch, Jr., G. L. Landgren, and S.T . Naumann. 1980. "Analytical Investigation of Dynamic Instability Occurring at Powerton Station." IEEE Trans. Pow. App. Sys. 99:1386-1395. 
Wang, L., and A. Semlyen. 1989. "Application of Sparse Eigenvalue Techniques to the Small Signal Stability Analysis of Large Power Systems." In Conference Papers: 1989 Power Industry Computer Application Conference, pp. 358-365. May 1-5 1989, Seattle, Washington. Institute of Electrical and Electronics Engineers, New York.

Wilkinson, J. A. 1965. The Algebraic Ejgenvalue Problem. Clarendon Press, 0xford.

Wong, D. Y., G. J. Rogers, B. Porretta, and P. Kundur. 1988. "Eigenvalue Analysis of Very Large Power Systems." IEEE Trans. Pow. Sys. 3:472-480. 


\section{APPENDIX A}

\section{CONVERSION OF MASS TO RUN ON A CRAY}


APPENDIX A

\section{CONVERSION OF MASS TO RUN ON A CRAY}

The 4000-bus VAX version of MASS from version 2.0 of the Small Signal Stability Program (SSSP) package was converted to run on a Cray. This appendix describes the changes that were made to allow MASS to run on a Cray. A file will be supplied to Bonneville, along with the Cray version of MASS, containing the results of using the VAX differences utility to compare the original MASS files and the final Cray versions of these files.

Only some sections of MASS were converted to run on the Cray. The Cray version of MASS 1) reads load flow data in Power Technologies Inc. (PTI) format only, 2) reads dynamic data in Ontario Hydro (OH) format only, and 3) does not allow the use of change case data. Furthermore, subroutine EIGENP, and the subroutines called by it, use a machine-dependent parameter TCON. This parameter was not modified for use on the Cray because subroutine EIGENP is not normally used.

The Cray FORTRAN 77 compiler CFT77 was used for this work. This compiter follows the FORTRAN 77 standard fairly closely. Thus, essentially all of the changes made to MASS were done to eliminate the use of nonstandard FORTRAN.

\section{GENERAL MODIFICATIONS TO MASS}

This section gives a general description of the modifications made to MASS.

The VAX version of MASS contained information in columns 73 and beyond. This caused the file containing MASS to be fairly large and unwieldy. Thus, all of the information in the columns beyond column 72 was deleted, and all trailing blanks were deleted from the resulting file. 0eleting the information in columns 73 and beyond will not change the performance of MASS because these columns are ignored by FORTRAN compilers. Deleting the trailing blanks changed a few of the output formats slightly, but the relevant format state- 
ments were modified to fix this. This modified version of the VAX version of MASS was the starting point for the changes described below.

The major things in the VAX version of MASS that had to be changed to make it run on a Cray were as follows:

- INTEGER ${ }^{2}$ and INTEGER $* 4$ statements were changed to INTEGER statements.

- Equivalencing of character variables to numeric variables was eliminated.

- Mixing of character and numeric data in common blocks was eliminated.

- Input/output files originally attached to logical units 5 and 6 were changed so that they would be attached to logical units 50 and 60 .

- Statements of the form "STOP 'Tong character string'" were replaced by the pair of statements "WRITE $\left({ }^{*},{ }^{*}\right)$ 'long character string" and "STOP".

- Calls to the vaX date utility were replaced by calls to the Cray date utiTity.

- Calls to vaX timing utilities were deleted.

Eliminating the use of INTEGER ${ }^{2}$ variables was the only change to MASS that required a significant amount of work.

Timing calls were added to some sections of MASS to allow the execution times of various subroutines to be determined. Timing calls were inserted by 1) writing a machine-dependent subroutine, GETCPU, which calls a machine timing utility, and 2) inserting calls to GETCPU at various places in MASS.

All of the subroutines used for 1) reading load flow data in formats other than PTI format, 2) reading dynamic data in formats other than $\mathrm{OH}$ format, or 3) doing change case computations were either deleted or replaced by dummy subroutines.

The INTEGER*2 statement in the section of FORTRAN code

COMMON / HORK / Jl(300), J2(600)

INTEGER $\star 2$ II $(600)$, I2(1200)

EQUIVALENCE (I1(1), J1(1)), (I2(1), J2(1)) 
cannot simply be changed to an INTEGER statement, because this would cause array Il to overlap array I2. In this situation, the dimension of $\mathrm{J} 1$ must be increased to 600 before the INTEGER*2 statement can be changed to an INTEGER statement. MASS makes extensive use of statements similar to those in the example above. This required that the dimension of the array GMWX be changed from NB*2 to NPL in subroutines FLODAl, DCLT SIMDAT, YSELF, YBRAN, RTRINT, CMBRAN, and SMIB.

MASS stores two INTEGER*2 variables in one INTEGER variable by using statements of the form

$$
\begin{aligned}
& \text { INTEGER*2 IARRAY(2) } \\
& \text { INTEGER I (I, IARRAY(1)). } \\
& \text { EQUIVALENCE ( }
\end{aligned}
$$

The effect of the above statements must be simulated when INTEGER 2 variables cannot be used. In particular, all of the information in two INTEGER variables, I1 and I2, must be packed into one INTEGER variable, INTOF2, without using INTEGER*2 variables. Furthermore, it must be possible to recover Il and I2 from INTOF2. The section of FORTRAN code

C Create a variable having all of the information contained

C in I1. Use bit 15 as a sign bit.

IF (I1 .LT. O) THEN

ITMP $=-I 1+2 * \star 15$

ELSE

ITMP $=$ II

ENDIF

C Store ITMP in bits $0-15$ of INTOF2, the absolute value of I2 in

$C$ bits 16-30, and give INTOF2 the same sign as I2.

IF (I2.LT. 0) THEN

ELSE

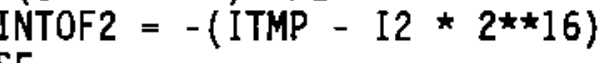

INTOF2 $=$ ITMP $+I 2 * 2 \star \star 16$

ENDIF

packs the integers 11 and I2 into the integer INTOF2, while the section of FORTRAN code

$$
\begin{aligned}
& \text { I2 = INTONE } / 2 \star \star 16 \\
& \text { IF (INTONE. LT. 0) THEN } \\
& \text { I1 }=- \text { INTONE }+ \text { I2 } 2 \star 2 \star \star 16 \\
& \text { ELSE } \\
& \text { I1 }=\text { INTONE - I2 } 2 * 2 \star 16
\end{aligned}
$$


C

\section{ENDIF}

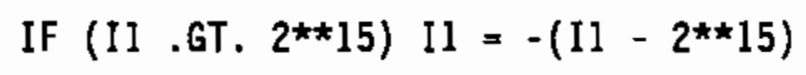

extracts $\mathrm{I} 1$ and $\mathrm{I} 2$ from INTOF2. These two algorithms are used by subroutines INTOF2 and SPLIT, respectively. The preceding code assumes that the magnitudes of I1 and I2 are sufficiently small that they would fit in an INTEGER*2 variable. This algorithm works because bits 0 through 14 of |II| and |I2| are nonzero, while bits 15 through 31 are zero. It was verified that INTOF2 and SPLIT work correctly on both a VAX and a Cray using the section of code

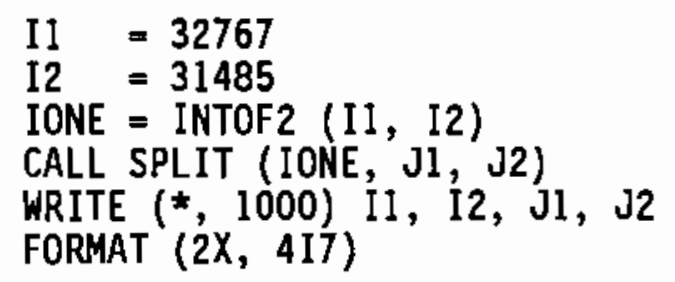

and verifying that $11=\mathrm{J} 1$ and $I 2=\mathrm{J} 2$. This was also done using $(I 1, I 2)=$ $(-32767,-31485),(376,-3145)$, and $(-67,1485)$, which represents all possible cases. CalTs to subroutines INTOF2 and SPLIT were incorporated into CMASS as required.

MASS stores character variables into integer variables using statements of the form

$\begin{array}{ll}\text { INTEGER } 2^{2} & \text { INAME } \\ \text { CHARACTER } 2^{2} & \text { CNAME } \\ \text { EQUIVALENCE } & \text { (INAME, CNAME) }\end{array}$

The effect of the above statements must be simulated because standard FORTRAN does not allow numeric and character data to be equivalenced. Thus, it is necessary to be able to write the data from a character variable into an integer variable, and to write the data in the integer variable back into a character variable. The FORTRAN code

\footnotetext{
CHARACTER $\star 8$ NULNAM, BIGNAM

C Initialize BIGNAM to the null string.

BIGNAM = NULNAM

C

C Left justify NAME(I: ISIZE) in BIGNAM. BIGNAM (I:ISIZE) = NAME (I:ISIZE)

READ (BIGNAM, ' $(A 4)^{\prime}$ ) INOFCH
} 
writes the first ISIZE characters of the character variable NAME into the loworder bits of the integer variable INOFCH. This code works on a VAX. On a Cray, the required code is

$$
\begin{aligned}
& \text { CHARACTER } 8 \text { NULNAM, BIGNAM } \\
& C \text { Initialize BIGNAM to the null string. } \\
& \text { BIGNAM = NULNAM } \\
& \text { C Right justify NAME( } 1 \text { : ISIZE) in BIGNAM. } \\
& \text { BIGNAM(9-ISIZE:8) = NAME (1: ISIZE) } \\
& \text { READ (BIGNAM, ' }(A B)^{\prime} \text { ) INOFCH }
\end{aligned}
$$

The preceding code represents the VAX and Cray versions, respectively, of subroutine INOFCH. MASS uses the variable INOFCH as one of the inputs to subroutine INTOF2. Thus, subroutine INOFCH must write the first ISIZE characters of NAME into the first ISIZE bytes of INOFCH and leave the higher-order bytes of INOFCH set to zero. Otherwise, subroutine INTOF2 will not work correctly when using INOFCH. The differences between the Cray and VAX versions of INOFCH are the result of differences in the way these machines write character variables into integer variables, which is not a standard FORTRAN 77 operation, and the fact that the Cray uses 64-bit words while the vaX uses 32-bit words. If the integer variable INAME is assigned a value by subroutine INOFCH, then the character equivalent of INAME, NAME, can be retrieved by the FORTRAN code

C

CHARACTER $* 8$ BIGNAM

$$
\begin{aligned}
& \text { WRITE (BIGNAM(1:4), '(A4)') INAME } \\
& \text { NAME (1:ISIZE) }=\text { BIGNAM(1:ISIZE) }
\end{aligned}
$$

on a VAX, or by the code

$$
\begin{aligned}
& \text { CHARACTER } * 8 \text { BIGNAM } \\
& \text { WRITE (BIGNAM, ' (A8)' }) \text { INAME } \\
& \text { NAME (1:ISIZE) }=\text { BIGNAM( }(9-\text { ISIZE:8) }
\end{aligned}
$$

on a Cray. The preceding sections of code are contained in the VAX and Cray version, respectively, of subroutine CHOFIN. It was verified that INOFCH and CHOFIN work correctly on both a VAX and a Cray by 1) running the FORTRAN code CHARACTER*4 NAME, NAME2 


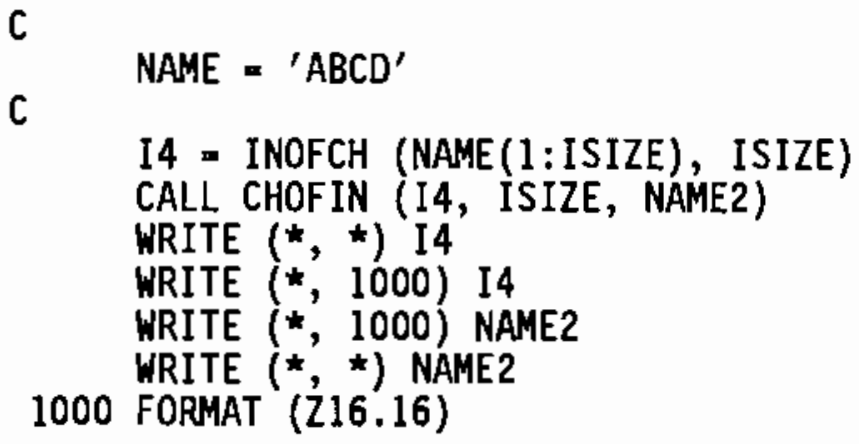

with ISIZE equal to one, two, three and four, 2) verifying that the variable NAME2 was equa t to NAME(1:ISIZE), and 3 ) verifying that bytes one to ISIZE of I4 were nonzero, while the higher-order bytes were all zero. The $Z$ format outputs variables in hexadecimal format and can be used to verify which bytes of a variable are zero. Calls to subroutines INOFCH and CHOFIN were incorporated into CMASS as required.

MASS calls the VAX DATE utility. Cray and VAX versions of a subroutine, GETDAY, was written. The VAX version of GETDAY simply calls the VAX date utility, while the Cray version of GETDAY calls the Cray date utility and converts the date from Cray format to VAX format. MASS's call to subroutine DATE was then replaced by a call to GETDAY.

The version of MASS that was created by making the changes described in this appendix consists of six files, CMASS.FOR, CMASS_VAX.FOR, CMASS_CRAY.FOR, CMASS_DUM.FOR, CMASS_EIS.FOR, and CMASS_OINV.FOR. The file CMASS.FOR contains most of the modified MASS subroutines. CMASS_VAX.FOR (CMASS_CRAY.FOR) contains the VAX- (Cray-) specific versions of subroutines INTOF2, SPLIT, INOFCH, CHOFIN, GETDAY, and GETCPU. CMASS_DUM.FOR contains a set of dummy subroutines that replace some of the subroutines deleted from the original version of MASS. CMASS_EIS.FOR contains the EISPAC (Smith et a1. 1976) eigenvalue subroutines that were in MASS. Finally, CMASS_OINV.FOR contains modified versions of the MASS subroutines INVERT and GAUS.

\section{SPECIFIC MODIFICATIONS OF MASS}

This section describes the changes made in individual MASS subroutines. Only changes that were not adequately covered in the preceding sections are described. 
Subroutine FLODAl

This section describes modifications made to subroutine FLODAl. The statements

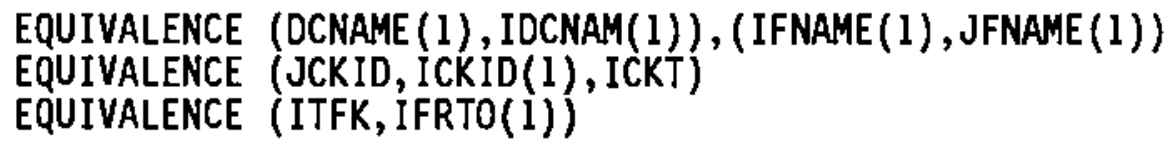

were replaced by

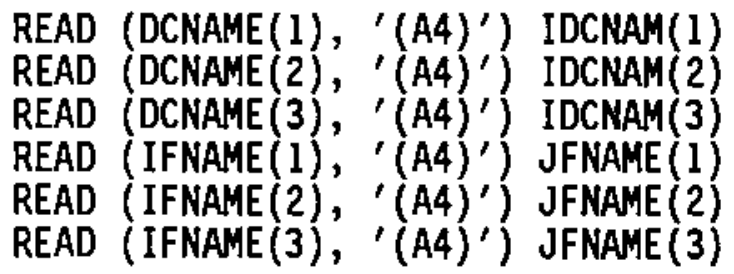

and, where necessary, statements of the form

ITFK = INTOF2 (IFRTO(1), IFRTO(2))

$\operatorname{ICKID}(1)=$ INOFCH $($ ICKT, 2)

JCKID = INTOF2 (ICKID(1), ICKID(2)).

Subroutine HEAD

This section describes modifications made to subroutine HEAD. The statements

EQUIVALENCE (JDATE, LDATE(1))

INTEGER $\approx 2$ IFRTO(2)

INTEGER $* 4$ ITFK

EQUIVALENCE (ITFK, IFRTO(1))

were replaced by

$$
\begin{aligned}
& \operatorname{LDATE}(1)=\operatorname{INOFCH}(\operatorname{JDATE}(1: 4), 4) \\
& \operatorname{LDATE}(2)=\operatorname{INOFCH}(\operatorname{JDATE}(5: 8), 4) \\
& \operatorname{LDATE}(3)=\operatorname{INOFCH}(\operatorname{JdATE}(9: 9), 1) .
\end{aligned}
$$

Furthermore, the statement

CALL DATEXX (JDATE, JTIME)

was replaced by

CALL DATEXX (JDATE)

because subroutine DATEXX has only one argument. 
Subrout ine YBRAN

This section describes modifications made to subroutine YBRAN. The statements

EQUIVALENCE (ITFK, IFRTO(1)), (JCKID, ICKT, JFRTO(1))

EQUIVALENCE (JCKID1, ICKT1)

were replaced by statements of the form

CALL SPLIT (JCKID, JFRTO(1), JFRTO(2))

CALL SPLIT (ITFK, IFRTO(1), IFRTO(2))

ITFK = INTOF2 (IFRTO(1), IFRTO(2))

CALL CHOFIN (JCKID1, 2, ICKT1)

as necessary. Furthermore, the statement

CALL HMOVE (0, IBRCH, IBRCH, NLFBUS)

was replaced by the statement

CALL MOVE(0, IBRCH, IBRCH, NLFBUS).

Subroutine MODAT

This section describes modifications made to subroutine MODAT. The statements

EQUIVALENCE (ITFK, IFRTO(1)), (JCKID, CKT, JARCCT(1))

CHARACTER*4 INETWK(6), NGRI, ISEM

were replaced by the statement

CHARACTER $\star 4$ INETWK (6), ISEM.

Furthermore, all of the code for doing change cases was deleted.

Subroutine PHEAD

This section describes modifications made to subroutine PHEAD. The statement

EQUIVALENCE (JDATE, LDATE(1))

was replaced by

CALL CHOF1N (LDATE(1), 4, JDATE(1:4))

CALL CHOFIN (LDATE (2), 4, JDATE $(5: 8)$ )

CALL CHOFIN (LDATE(3), 1, JDATE(9:9)). 
Subroutine GENINT

This section describes modifications made to subroutine GENINT. The statements

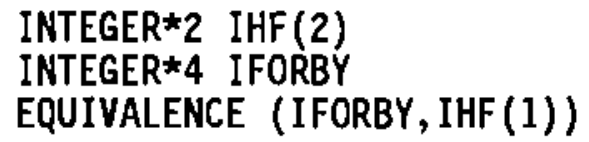

were deleted. Furthermore, the statements

1700 FORMAT $(/ /, I X, '$ SATURATION PARAMETERS $: ', / /, 1 X, '$ TYPE $=$ $+\prime, \mathrm{I} 2, /, 2 \mathrm{X},{ }^{\prime} \mathrm{ASAT}={ }^{\prime}, \mathrm{F} 6.3,7 \mathrm{X}, \mathrm{BSSAT}^{\prime}{ }^{\prime}, \mathrm{F} 6.3, \mathrm{BX},{ }^{\prime}$ BATL $={ }^{\prime}, \mathrm{F} 6.3,4 \mathrm{X},{ }^{\prime} \mathrm{BAT}$

1720 FORMAT $/, 1 X,{ }^{\prime} M E C H A N I C A L$ PARAMETERS $: ', / /, 1 X, ' \quad H M=\prime, F B .3,7 X, ' \mathrm{KD}$ $\left.+={ }^{\prime}, \mathrm{E} 10.4,6 \mathrm{X},{ }^{\prime} \mathrm{CSG}={ }^{\prime}, \mathrm{F} 6.3,5 \mathrm{X},{ }^{\prime} \mathrm{CGG}={ }^{\prime}, \mathrm{F} 6.3\right)$

were replaced by

1700 FORMAT $(/ /, 1 X$, SATURATION PARAMETERS $: ', / /, 1 X, '$ TYPE $=\prime, 13 X$,

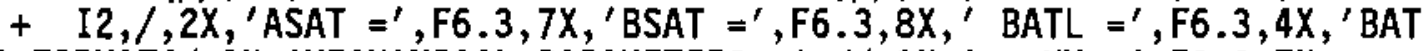

1720 FORMAT $(/, 1 X$, MECHANICAL PARAMETERS $: ', / /, 1 X, ' \quad H M=\prime, F 8.3,7 X$, $\left.+' \mathrm{KD}=\prime, \mathrm{E} 10.4,6 \mathrm{X},{ }^{\prime} \mathrm{CSG}={ }^{\prime}, \mathrm{F} 6.3,5 \mathrm{X},{ }^{\prime} \mathrm{CGG}={ }^{\prime}, \mathrm{F} 6.3\right)$.

Subroutine AEIGN

This section describes modifications made to subroutine AEIGN. The statements

INTEGER*2 JORD(NSI), KORD(NSI), LORD (NSI)

EQUIVALENCE (IBUF (1), IBUFI (1)), (IBUF2(1),V1(1))

EQUIVALENCE (CIK, IK)

were deleted.

Subroutine DPLT

This section describes modifications made to subroutine DPLT. The statements

CHARACTER $* 4$ JDATE (3)

CALL CHOFIN (LDATE(1), 4, JDATE(1))

CALL CHOF IN (LDATE (2), 4, JDATE (2))

CALL CHOFIN (LDATE(3), I, JDATE(3) (1:1))

were inserted, and each occurrence of the statement

WRITE(IU6, 450) LFNO, KASE, (ITEXT(LK), LK=1, 20), LDATE, (ISWTEX(LL), LL=

was replaced by the statement 
WRITE(IU6, 450) LFNO, KASE, (ITEXT(LK), LK=1, 20), JDATE, (ISWTEX(LL), LL= . Subroutine DPFLT

This section describes modifications made to subroutine DFPLT. The statements

CHARACTER $* 4$ JDATE $(3)$

CALL CHOF IN (LDATE(1), 4, JDATE(1))

CALL CHOFIN (LDATE(2), 4, JDATE(2))

CALL CHOF IN (LDATE(3), 1, JDATE(3)(1:1))

were inserted, and each occurrence of the statement

WRITE(IU6,680) LFNO, KASE, (ITEXT(LK), LK=1, 20), LDATE,

was replaced by the statement

WRITE(IU6, 680) LFNO, KASE, (ITEXT(LK), LK=1, 20), JDATE, .

\section{Main Program MASS}

This section describes modifications made to the main program MASS. The one-dimensional arrays SCRATO, SCRAT1, and SCRAT4 were initially of size $N B+N P L * 5, N B+12 * N P L$, and 50,000 , respectively. These sizes were changed to $N B+N P L * 6,4 * N B+12 * N P L$, and 100,000 , respectively.

Subroutine DGEDAT

This section describes modifications made to subroutine DGEDAT. The name of the variable NGRI in common block PBK was changed to INGRI since NGRI is declared to be a character variable. Statements of the form

INGRI = INOFCH (NGRI, 4),

were inserted as necessary.

Subroutines READAT, DCLAT

This section describes modifications made to subroutines READAT and DCLDAT. The statement

EQUIVALENCE (ICN, ICKT)

was deleted, and statements of the form

ICN = INOFCH (ICKT, 2) 
were inserted as necessary.

Subroutine BANNER

This section describes modifications made to subroutine BANNER. The format statement labeled 1 was split into two format statements, and additional WRITE statements were added as necessary.

Subroutine DATEXX

This section describes modifications made to subroutine DATEXX. The statement

CALL DATE (VDATE)

was replaced by the statement

CALL GETDAY (VDATE).

Subroutine AGEN

This section describes modifications made to subroutine AGEN. The names of the variables DPGSUM1, DPGSUM2, and DPGRSUM were changed to DPGSM1, DPGSM2, and DPGRSM, respectively.

Subroutine AECON

This section describes modifications made to subroutine AECON. The statements

INTEGER 2 IHF(2)

INTEGER*4 IFORBY

EQUIVALENCE (IFORBY, IHF(1))

were deleted.

\section{Subroutine STBDTA}

This section describes modifications made to subroutine BLOCK DATA STBDTA. The data statements assigning the values 5, 6 , and 6 to IU5, IU6, and IUD6, respectively, were changed so that these variables were assigned the values 50,60 , and 60 , respectively. 

APPENDIX B

IESTING THE CRAY VERSION OF MASS 
APPENDIX B

\section{IESTING THE CRAY VERSION OF MASS}

This appendix describes the tests that were run to verify that the cray version of MASS works correctly.

The Cray version of MASS, CMASS, consists of the files CMASS.FOR, CMASS_CRAY.FOR, CMASS_DUM.FOR, CMASS_EIS.FOR, and CMASS_INV.FOR. CMASS a7 so

calls the function ISAMAX in the Cray scientific library. CMASS_CRAY.FOR contains subroutines that are machine-dependent, while all of the other files contain subroutines that should run on most machines with a standard FORTRAN 77 compiler. CMASS can be run on a VAX by replacing the subroutines in CMASS_CRAY.FOR with the subroutines in CMASS_VAX.FOR and using ISAMAX.FOR in place of the corresponding Cray scientific library function. ISAMAX.FOR contains the LINPACK subroutine ISAMAX, while CMASS_INV.FOR contains the LINPACK subroutines used to compute the inverse of the matrix of right eigenvectors. An alternative Cray version of MASS can be obtained by replacing the file CMASS_INV.FOR with the file CMASS_OINV.FOR, which contains the subroutines used by the original VAX version of MASS to compute the inverse of the matrix of right eigenvectors. Using CMASS_OINV.FOR instead of CMASS_INV.FOR will change the results computed by CMASS slightly. The subroutines in CMASS_OINV.FOR have not been tested on the Cray, and are not optimized for use on the cray. The changes needed to optimize CMASS_OINV.FOR for the Cray are described in Section 5.

The files CMASS.FOR, CMASS_CRAY.FOR, CMASS_VAX.FOR, CMASS_DUM.FOR, and CMASS_OINV.FOR are identical to the corresponding files described in Appendix A. CMASS_INV.FOR was obtained by modifying CMASS_OINV.FOR to use the LINPACK matrix inversion routines described in Section 5. Finally, CMASS_EIS. FOR was obtained by making the modifications described in Section 5 to the EISPACK subroutines in the version of this file that was described in Appendix $A$.

Six test problems, which will be called the 9BUS, 11BUS, 39BUS, 39BUSSVC, 39BUSRES and T3500 problems, were supplied with the VAX version of MASS. 
CMASS was tested using only these problems. These problems do not exercise all of the options in MASS. Hence, the testing described below should not be viewed as being comprehensive.

The first VAX test of CHASS was designed to verify that MASSV2-4K, the 4000-bus VAX version of MASS supplied by EPRI, worked correctiy. This test consisted of I) compiling the EPRI-supplied version of MASS, MASSV2-4K, 2) running each of the test problems using MASSV2-4K, and 3) using the VAX differences utility to verify that the results produced by MASSV2-4K were identical, except for run dates, to the reference output files supplied with these test problems.

The second VAX test of CMASS was designed to verify that CMASS_VAX_OINV, obtained by compiling and linking the files CMASS.FOR, CMASS_VAX.FOR, CMASS_DUM.FOR, CMASS_EIS.FOR, CMASS_OINV.FOR, and ISAMAX.FOR on a VAX, computes exactly the same results as MASSV2-4K. Note that CMASS_VAX_OINV is almost the same as CMASS, the Cray version of MASS. This test consisted of 1) running each test problem using CMASS_VAX_OINV and MASSV2-4K, and 2) using the VAX differences utijity to verify that the results produced by CMASS_VAX_OINV and MASSV2-4K were identical.

The third VAX test of CMASS is an extension of the second VAX test. The test problems do only a limited amount of output when using the data files supplied with the SSSP package. Therefore, the third VAX test consisted of 1) modifying the PRINT SPECIFICATION data files for the gBUS, 11BUS, 39BUS, 39BUSSVC and 39BUSRES test problems so that MASS would output the state matrix, participation vectors, and left and right eigenvectors; 2) running MASSV2-4K and CMASS_VAX_OINV using these modified data files; and 3) using the VAX differences utility to verify that both codes produced exactly the same results. The T3500 problem was not used in this test because it would produce an extremely large output file.

The fourth VAX test of CMASS was designed to verify that CMASS_VAX_INV, obtained by compiling and linking the files CMASS.FOR, CMASS_VAX.FOR, CMASS_DUM.FOR, CMASS_EIS.FOR, CMASS_INV.FOR, and ISAMAX.FOR on a VAX, computes essentially the same results as MASSV2-4K. The only difference between CMASS_VAX_INV and CMASS is that CMASS_VAX_INV uses the VAX-specific 
subroutines in CMASS VAX.FOR, while CMASS uses the Cray-specific subrout ines in CMASS_CRAY.FOR. In theory, the state matrix, eigenvalues, and right eigenvectors computed by CMASS_VAX_INV should be identical to the values computed by CMASS_VAX_OINV because these computations are not affected by the method used to invert the matrix of right eigenvectors. Furthermore, the left eigenvectors computed by CMASS_INV.FOR should be slightly different from the results computed by CMASS_OINV.FOR because of differences in roundoff errors in the algorithms used by the subroutines in these two files. Finally, the participation vectors and frequency responses computed by these codes should be slightly different because they depend upon the values of the left eigenvectors.

The fourth vaX test of MASS was conducted as follows. Each test problem was run using CMASS_VAX_INV, and the VAX differences utility was used to find a11 of the differences between the results produced by CMASS_VAX_INV and MASSV2-4K. It was found that the state matrices, eigenvalues, participation vectors, and right eigenvectors computed by CMASS_VAX_INV, at least those printed out by the test problems, were identical to the values computed by CMASS_VAX_OINV. In fact, the only differences between the results of CMASS_VAX_INV and CMASS_VAX_OINV were as follows:

- The left eigenvectors were slightly different in the 39BUS case, the greatest difference being about 0.001 based on random examination of differences.

- The frequency response results for the 39BUSRES problem were slightly different, the greatest difference being about 0.003 based on random examination of differences.

- The dominant state, which is determined by the participation vector, changed from "generator speed" to "generator angle," or vice versa, for several eigenvalues in the $T 3500$ problem.

These results are consistent with roundoff differences in the algorithms in CMASS_OINV.FOR and CMASS_INV.FOR. The fact that no differences were detected in the actual participation vectors computed by the two codes is probably because MASS outputs only the first few digits of the participation vectors. This test provides only a limited amount of information because most of the 
test problems print only eigenvalues and participation vectors. A more quantitative version of this test is described below.

After the preceding tests were completed, the files CMASSX.FOR and CMASS_Tl.FOR were created. CMASSX.FOR is the same as CMASS.FOR, except that subroutines AEIGN and REIGEN have been deleted. Subroutines AEIGN and REIGEN were placed in the file CMASS_Tl.FOR and modified so that 1) subroutine REIGEN prints the state matrix to a dump file and 2) subroutine AEIGN prints the right and left eigenvectors and the participation vectors to the dump file. These results were output using a 1 PE 16.8 format. Thus, the first nine digits of each result were printed. A third file, CMASS_T2.FOR, was created by modifying CMASS_T1.FOR so that it opens the dump file as a binary file.

The fifth VAX test of CMASS is a quantitative version of the fourth VAX test. This test was conducted as follows. CMASS_TI_OINV was generated by compiling and linking CMASSX.FOR, CMASS_Tl.FOR, CMASS_VAX.FOR, CMASS_DUM.FOR, CMASS_EIS.FOR, CMASS_OINV.FOR, and ISAMAX.FOR on a VAX, and CMASS_T1_INV was generated by compiling and linking CMASSX.FOR, CMASS_Tl.FOR, CMASS_VAX.FOR, CMASS_DUM.FOR, CMASS_EIS.FOR, CMASS_INV.FDR, and ISAMAX.FOR on a VAX. CMASS_TI_OINV is the same as CMASS_VAX_OINV, except that CMASSX.FOR and CMASS_T1 were used in place of CMASS.FOR. Similarly, CMASS_TI_INV is the same as CMASS_VAX_INV, except that CMASSX.FOR and CMASS_T1 were used in place of CMASS.FOR. Each of the test problems, except for the T3500 problem, was then run using CMASS_T1_OINV and CMASS_T1_INV. The modified PRINT SPECIFICATION data files described in the third VAX test were used in this test. The preceding was repeated for the T3500 problem, but CMASS_T2.FOR was used in place of CMASS_T1.FOR when generating the executable code used to run the T3500 problem. The VAX differences utility was used to verify that the standard output files created by CMASS_Tl_OINV were identical to the files created by MASSV2-4K. Furthermore, random entries in the dump file created by CMASS_T1_0INV were compared to the results in the standard MASS output file to verify that the correct data were printed to the dump files. This was done only for the gBuS problen.

MASS generates an $\mathrm{N}$ by $\mathrm{N}$ real matrix of left eigenvectors, where $\mathrm{N}$ is the number of states in the test problem. Let $L_{i, j}$ be the entry in the $i$ th row 
and $j$ th column of the left eigenvector matrix. If the ith eigenvalue is real, then $L E_{i, j}$ is equal to the $j$ th entry in the left eigenvector corresponding to the $i$ th eigenvalue. If eigenvalues $i$ and $i+1$ are complex conjugates, then $L E_{j, j}$ is equal to the real part of the $j$ th entry in the left eigenvector corresponding to eigenvalues $i$ and $i+1$, while $L E_{i+1, j}$ is equal to the imaginary part of the $j$ th entry in the left eigenvector corresponding to eigenvalues $i$ and $i+1$. Let $L E_{i, j}$ and $O L E_{i, j}$ be the entries in the left eigenvector matrices computed when using CMASS_INV.FOR and CMASS_OINV.FOR, respectively. A program was written to compute the quantities

$$
\begin{aligned}
& \underset{i, j}{\operatorname{Maximum}\{\text { Minimum }}\left(\left|L E_{i, j}-O L E_{i, j}\right|,\left|L E_{i, j}-O L E_{i, j}\right| /\left|O L E_{i, j}\right|\right\} \text {, } \\
& \underset{i, j}{\operatorname{Maximum}}\left|L E_{i, j}-O L E_{i, j}\right|, \\
& \underset{i, j}{\text { Average }}\left|L E_{j, j}-O L E_{i, j}\right|
\end{aligned}
$$

and

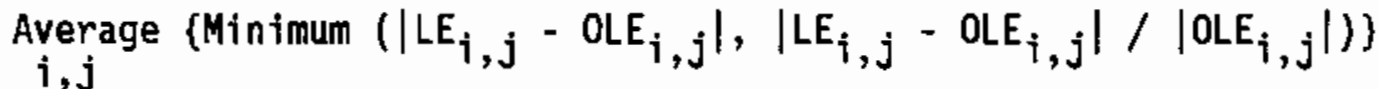

using the data in the dump files created by the programs described above. If $O L E_{i, j}=0$, then $\left|L E_{i, j}-O L E_{i, j}\right| /\left|O L E_{i, j}\right|$ is replaced by $\left|L E_{i, j}-O L E_{i, j}\right|$ in the preceding. The results of the preceding computations are listed in columns 2 through 5, respectively, of Table B.1. Similar computations were done for the states matrices, eigenvalues, right eigenvectors, and participation vectors. The participation vector results are 1isted in Table B.2, while no differences were found when comparing the states matrices, eigenvalues, or right eigenvectors.

The results in columns 4 and 5 of Table B.l show that the results computed when using CMASS_INV.FOR are essentially the same as the results computed when using CMASS_OINV.FOR. Furthermore, the facts that 1) for each test problem the average value of the entries in the left eigenvector matrix is in the range of 0.17 to 2.71 and 2) the relative precision of the VAX is approximately $10^{-7}$ indicate that the average differences between the left 
TABLE B.1. Differences in Left Eigenvectors

\begin{tabular}{|c|c|c|c|c|}
\hline coblem & Max. Min. & Max. Abs. & Ave. Abs. & Ave. Min. \\
\hline 9BU & $3.5 \times 10^{-5}$ & $1.1 \times 10^{-4}$ & $2.0 \times 10^{-6}$ & $1.2 \times 10^{-6}$ \\
\hline 11BUS & $4.6 \times 10^{-4}$ & $7.6 \times 10^{-4}$ & $6.4 \times 10^{-6}$ & $3.2 \times 10^{-6}$ \\
\hline 39BUS & $8.0 \times 10^{-5}$ & $8.0 \times 10^{-5}$ & $4.7 \times 10^{-7}$ & $4.2 \times 10^{-7}$ \\
\hline 398USSVC & $1.1 \times 10^{-4}$ & $1.1 \times 10^{-4}$ & $4.2 \times 10^{-7}$ & $3.8 \times 10^{-7}$ \\
\hline RES & $8.0 \times 10^{-5}$ & $8.0 \times 10^{-5}$ & $4.7 \times 10^{-7}$ & $4.2 \times 10^{-7}$ \\
\hline $\mathrm{T} 3500$ & $6.5 \times 10^{-2}$ & $6.5 \times 10^{-2}$ & $5.7 \times 10^{-6}$ & $4.4 \times 10^{-6}$ \\
\hline
\end{tabular}

IABLE B.2. Differences in Participation Vectors

\begin{tabular}{|c|c|c|c|c|}
\hline$\frac{\text { est Problem }}{\text { gBUS }}$ & $\frac{\text { Max.Min. }}{8.9 \times 10^{-6}}$ & $\frac{\text { Max. Abs. }}{8.9 \times 10^{-6}}$ & $\frac{\text { Ave. Abs. }}{1.8 \times 10^{-7}}$ & $\frac{\text { Ave. Min. }}{1.8 \times 10^{-7}}$ \\
\hline 1. & $5.9 \times 10^{-5}$ & $5.9 \times 10^{-5}$ & $2.0 \times 10^{-7}$ & $2.0 \times 10^{-7}$ \\
\hline $39 \mathrm{BUS}$ & $3.2 \times 10^{-6}$ & $3.2 \times 10^{-6}$ & $1.7 \times 10^{-8}$ & $1.7 \times 10^{-8}$ \\
\hline SVC & $5.2 \times 10^{-6}$ & $5.2 \times 10^{-6}$ & $2.0 \times 10^{-8}$ & $2.0 \times 10^{-8}$ \\
\hline 39BUSR & $3.2 \times 10^{-6}$ & $3.2 \times 10^{-6}$ & $1.7 \times 10^{-8}$ & $1.7 \times 10^{-8}$ \\
\hline T3500 & $3.6 \times 10^{-5}$ & $3.6 \times 10^{-5}$ & $8.0 \times 10^{-9}$ & $8.0 \times 10^{-9}$ \\
\hline
\end{tabular}

eigenvectors computed using the algorithms in CMASS_OINV.FOR and CMASS_INV.FOR are attributable to differences in the roundoff behavior of these algorithms. The maximum differences listed in Table B.1 for the first five test problems can also be attributed to differences in the roundoff behavior of the algorithms in CMASS_OINV.FOR and CMASS_INV.FOR.

The maximum differences listed in Table B.1 for the T3500 test problem are significantly larger than the corresponding results for the other five problems. It was verified that the moderately large differences in the left eigenvectors for the T3500 problem are due to roundoff errors as follows. A variant of CMASS_T1_INV, CMASS_T6_INV, was created which output the left and right eigenvector matrices to a binary file. A test program, TSTINV, was then written which read in the right eigenvector matrix and computed its inverse using the LINPACK subroutines SGEFA and SGEDI (Dongarra et al. 1979). It was found that the left eigenvectors computed by TSTINV were identical to the results computed by CMASS_T6_INV. This strongly indicates that the modified 
versions of SGEFA and SGEDI in CMASS_INV.FOR work correctiy. A second test program computed the "exact" left eigenvectors by 1) reading in the right eigenvector matrix, 2) converting it to double precision, and 3) computing its inverse using double-precision versions of SGEFA and SGEDI. The results of comparing the "EXACT" left eigenvectors with the results computed when using CMASS_INV.FOR and CMASS_OINV.FOR are Tisted in Table B.3. The data in this table show that the accuracy of the results computed using CMASS_INV.FOR is essentially the same as the accuracy of the results computed using CMASS OINV.FOR. Finally, using the LINPACK subroutine SGECO, it was found that the condition number of the matrix of right efgenvectors is approximately equal to $10^{6}$. This condition number indicates that for some choices of $b$, the computed solution of $A x=b$ will have only about $k$ - 6 correct decimal digits, where $k$ is the number of decimal digits in a computer word (Golub and Van Loan 1983, p. 72). This indicates that some entries in the left eigenvector matrix for the $T 3500$ problem may have only one significant digit because $k$ is approximately equal to 7 for single-precision arithmetic on a VAX. This is consistent with the results shown in Table B.3. This problem shows that it would be useful for MASS to compute and print the condition number of the matrix of right eigenvectors. Furthermore, it shows that, for some problems, the use of double precision on a VAX will be necessary to obtain accurate left eigenvectors.

Table B. 2 shows that the differences between the participation vectors computed when using CMASS_INV.FOR and when using CMASS_OINV.FOR are consistent with the differences in the left eigenvectors.

The preceding tests indicate that the changes made in deriving the files CMASS.FOR, CMASS_VAX.FOR, CMASS_DUM.FOR, CMASS_EIS.FOR and CMASS_OINV.FOR from

TABLE B.3. Errors in Left Eigenvectors

\begin{tabular}{|c|c|c|c|c|}
\hline 6 & x. Min. & ax. Abs. & Ave. Abs. & Ave \\
\hline & & 3 & $4.6 \times 10^{-t}$ & $3.3 \times 10$ \\
\hline (ACT & $3.6 \times 10^{-2}$ & $3.9 \times 10^{-2}$ & $4.8 \times 10^{-6}$ & $3.4 \times 10^{-6}$ \\
\hline
\end{tabular}


the original VAX version of MASS, MASSV2-4K.FOR, did not change the performance of MASSV2-4K. Furthermore, the preceding tests show that CMASS_INV.FOR and ISAMAX.FOR work correctly when used in place of CMASS_OINV.FOR. The remaining tests are to verify that CMASS, consisting of the files CMASS.FOR, CMASS_CRAY.FOR, CMASS_DUM.FOR, CMASS_EIS.FOR, CMASS_INV.FOR, and the Cray scientific library function, ISAMAX, work correctly on a Cray. In the following, CMASS_VAX will be used to denote the VAX-executable code consisting of CMASS.FOR, CMASS_VAX.FOR, CMASS_DUM.FOR, CMASS_EIS.FOR, CMASS_INV.FOR, and ISAMAX.FOR.

The only differences between CMASS and CMASS_vAX are that 1) CMASS uses the subroutines in the file CMASS_CRAY.FOR, while CMASS_VAX uses the subroutines in the file CMASS_VAX.FOR; 2) CMASS uses the Cray scientific library function ISAMAX, while CMASS_VAX uses the subroutine in the file ISAMAX.FOR; and 3) CMASS is run on a Cray, while CMASS_VAX is run on a VAX. Thus, the main factors that might cause CMASS to work incorrectly while CMASS_VAX works correctly are 1) the subroutines in CMASS_CRAY.FOR do not give the correct results on the Cray, while the corresponding subroutines in CMASS_VAX.FOR give the correct results on the VAX; 2) the Cray scientific 1 ibrary subroutine ISAMAX does not produce the same results as ISAMAX.FOR; and 3) differences between the Cray and VAX FORTRAN compilers cause CMASS to produce incorrect results while CMASS_VAX produces correct results. It should be kept in mind that the results produced by CMASS should be more accurate than the results produced by CMASS_VAX because the Cray uses 64-bit arithmetic, while the VAX uses 32-bit arithmetic.

Appendix A describes the tests that were done to verify that the subroutines in CMASS_CRAY.FOR work correctly. It is assumed that any incompatibilities between ISAMAX.FOR and the corresponding Cray scientific library function would be detectable as errors in left eigenvectors computed by CMASS. Thus, no direct testing was done to verify that ISAMAX.FOR and the Cray scientific library version of ISAMAX produce the same results. Thus, the tests described below are general tests designed to verify that CMASS works correctiy when compiled and run on a cray. 
The first Cray test of CMASS was intended to verify that each of the test problems will run to completion using CMASS. This test was conducted by running each of the test problems on the cray using CMASS. Some of the test problems caused CMASS to fail. It was detemined that some of the subroutines in MASS use uninitialized variables and assume that these variables have a value of zero. This assumption is not valid on the cray unless the code is compiled with the "-a static" option. Therefore, CMASS was recompiled using the "-a static" option and all of the test problems were rerun. This time all of the problems ran to completion.

The second Cray test of CMASS was intended to verify that the results obtained for each of the test problems were essentially the same when computed using CMASS and CMASS_VAX. This test was conducted as follows. CMASSX_CRAY was created by compiling CMASSX.FOR, CMASS_T1.FOR, CMASS_CRAY.FOR, CMASS_DUM.FOR, CMASS_EIS.FOR, and CMASS_INV.FOR on the Cray. CMASSX.FOR and CMASS_T1.FOR were described above. Al1 of the test problems, excluding the T3500 problem, were then run using CMASSX_CRAY. The Cray differences utility was used to verify that the standard MASS output files created by CMASS and CMASSX_CRAY were identical. Next, the dump files created by CMASSX_CRAY were compared with the dump files created by CMASS_Tl_INV, which was described above. The results of these comparisons are listed in Tables B.4 through B.7. The results for the participation vectors are not listed, but are similar to the results given in Table B.7 for the left eigenvectors. The first five columns in Tables B.4 through B.7 have the same meanings as the columns in Table B.1, while the sixth column is the average value of the elements of the appropriate matrix.

A variant of CMASSX_CRAY was used to run the T3500 problem and print the state matrix for this problem. This state matrix was compared to the state matrix output by a variant of CMASS_VAX. The results of this comparison are listed in Table B.4.

The results in Table B.4 show that the state matrices generated by CMASS and CMASS_VAX are nearly the same. Furthermore, for each test problem the average "relative difference" in the entries of the state matrices computed by CMASS and CMASS_VAX are in the range $2.5 \times 10^{-7}$ to $2.8 \times 10^{-6}$, which is about the 
TABLE B.4. Differences in States Matrices $\frac{\text { Iest Problem }}{\text { gBuS }} \frac{\text { Max. Min. }}{4.1 \times 10^{-5}} \frac{\text { Max.Abs. }}{3.4 \times 10^{-4}} \frac{\text { Ave. Abs. }}{4.1 \times 10^{-6}} \frac{\text { Ave. Min. }}{6.8 \times 10^{-7}} \frac{\text { Ave. Ele. }}{2.1}$

$$
1.6 \times 10^{-5} \quad 4.9 \times 10^{-4}
$$

$3.3 \times 10^{-6}$

$2.6 \times 10^{-7}$

6.4

39BUS

$3.3 \times 10^{-6}$

$353 \times 10^{-5}$

$1.3 \times 10^{-7}$

$3.9 \times 10^{-8}$

0.39

39BUSSVC $2.9 \times 10^{-6}$

$5.3 \times 10^{-5}$

$1.2 \times 10^{-7}$

$3.1 \times 10^{-8}$

0.40

39BUSRES

$3.2 \times 10^{-6}$

$5.3 \times 10^{-5}$

$1.3 \times 10^{-7}$

$3.9 \times 10^{-8}$

0.39

T 3500

$5.3 \times 10^{-2}$

$5.8 \times 10^{1}$

$2.7 \times 10^{-4}$

$1.2 \times 10^{-6}$

0.61

IABLE B.5. Differences in Eigenvalues

\begin{tabular}{|c|c|c|c|c|c|}
\hline Iest Problem & Max. Min. & Max. Abs. & Ave, Abs. & Ave. Min & Ave. Ele. \\
\hline gBUS & $8.8 \times 10^{-4}$ & $8.8 \times 10^{-4}$ & $3.6 \times 10^{-5}$ & $3.1 \times 10^{-5}$ & 4.7 \\
\hline 11BUS & $9.1 \times 10^{-5}$ & $1.8 \times 10^{-4}$ & $2.6 \times 10^{-5}$ & $9.2 \times 10^{-6}$ & 9.3 \\
\hline 39BUS & $1.1 \times 10^{-5}$ & $6.9 \times 10^{-5}$ & $7.3 \times 10^{-6}$ & $1.7 \times 10^{-6}$ & 3.1 \\
\hline 39BUSSVC & $1.3 \times 10^{-5}$ & $8.8 \times 10^{-5}$ & $7.1 \times 10^{-6}$ & $2.2 \times 10^{-6}$ & 3.1 \\
\hline 39BUSRES & $1.1 \times 10^{-5}$ & $6.9 \times 10^{-5}$ & $7.3 \times 10^{-6}$ & $1.7 \times 10^{-6}$ & 3.1 \\
\hline
\end{tabular}

IABLE_B.6. Differences in Right Eigenvectors

Test Problem

9BUS

11BUS

39BUS

39BUSSVC

39BUSRES
Max. Min.

$$
1.2 \times 10^{-3}
$$

$5.6 \times 10^{-4}$

$3.9 \times 10^{-2}$

$3.4 \times 10^{-2}$

$3.9 \times 10^{-2}$ $\frac{\text { Max. Abs. }}{1.2 \times 10^{-3}}$

$$
5.6 \times 10^{-4}
$$$$
4.9 \times 10^{-2}
$$

$4.6 \times 10^{-2}$

$4.9 \times 10^{-2}$
Ave. Abs.

$7.8 \times 10^{-6}$

$7.9 \times 10^{-6}$

$1.4 \times 10^{-5}$

$1.4 \times 10^{-5}$

$1.4 \times 10^{-5}$ $\frac{\text { Ave. Min. }}{7.7 \times 10^{-6}}$

$7.8 \times 10^{-6}$

$1.3 \times 10^{-5}$

$1.2 \times 10^{-5}$

$1.3 \times 10^{-5}$
Ave. Ele.

0.15

0.10

0.10

0.10

0.10

IABLE B.7. Differences in Left Eigenvectors

Test Problem gBus

IIBUS

39BUS

39BUSSVC

39BUSRES
Max. Min

$$
2.2 \times 10^{-2}
$$

$8.4 \times 10^{-3}$

$8.7 \times 10^{-3}$

$7.7 \times 10^{-3}$

$8.7 \times 10^{-3}$ $\frac{\text { Max. Abs. }}{2.3 \times 10^{0}}$

$3.4 \times 10^{-2}$

$8.7 \times 10^{-3}$

$7.7 \times 10^{-3}$

$8.7 \times 10^{-3}$ $\frac{\text { Ave. Abs. }}{1.3 \times 10^{-2}}$

$1.9 \times 10^{-4}$

$1.8 \times 10^{-5}$

$2.0 \times 10^{-5}$

$1.8 \times 10^{-5}$
Ave. Min. Ave. Ele.

$6.8 \times 10^{-4} \quad 1.3$

$3.4 \times 10^{-5}$

$1.4 \times 10^{-5}$

1. $5 \times 10^{-5}$

$1.4 \times 10^{-5}$
2.7

0.17

0.17

0.17 
best that can be expected because the VAX has a relative precision of about $10^{-7}$. The results for the first five test problems in Table B.4 indicate that most of the subroutines required to read in the problem data and generate the state matrix are working correctly.

The "maximum absolute difference" listed in Table B.4 for the T3500 problem, 58, corresponds to a relative difference of about 0.02 and thus is not as bad as it appears. The relatively large "maximum differences" for the T3500 problem, however, are larger than one would like to see. Two major factors could be the cause of these large differences. If a few of the entries in the $T 3500$ problem state matrix are very sensitive to roundoff errors, then these differences could be the result of the fact that the cray uses 64-bit words, while the VAX uses 32-bit words. If the differences in the state matrices are not the result of roundoff errors, then it is likely that there is a bug in CMASS. Determining the actual cause of the differences in the $T 3500$ state matrices will probably be fairly time-consuming and has not been pursued because of time limitations. It is strongly recommended, however, that the cause of these differences be determined before the Cray version of MASS is used for the analysis of power systems. Note that if a double-precision VAX version of MASS is available, then it could be used to determine if the differences in the state matrices are the result of roundoff errors.

Tables B.5 through B.7 show that the eigenvalues, right eigenvectors, and left eigenvectors computed by CMASS_VAX and CMASS are essentially the same and that the differences are reasonably attributabie to roundoff errors.

No data for the $T 3500$ problem are included in Tables B.5 through B.7. This is because the differences in the state matrices computed by CHASS and CMASS_VAX cause the eigenvalues to be slightly different. The differences are large enough that the ith eigenvalue computed by CMASS should be associated with the $j$ th eigenvalue computed by CMASS_VAX, where $j \neq j$. Thus, writing a program to compute the quantities in Tables B.5 through B.7 would be fairly difficult for the $T 3500$ problem. Instead, the VAX differences utjitity was used to compare the eigenvalues in the standard output files generated by CMASS_VAX and CMASS. It was found that I) in most cases, the ith eigenvalue 
computed by CMASS was the same, to several significant digits, as the ith eigenvalue computed by CMASS_VAX; 2 ) in most other cases, the $i$ th eigenvalue computed by CMASS was the same or similar to the kth eigenvalue computed by CMASS_VAX where $|i-k|$ is greater than zero, but smal1; 3) CMASS_VAX computed the pair of complex eigenvalues $-31.78 \pm j 0.023$, while CMASS computed the two real eigenvalues -31.57 and -31.80 ; 4) CMASS_VAX computed the eigenvalue 30.28, which does not appear to be associated with any eigenvalue computed by CMASS; and 5) CMASS computed the eigenvalue -39.22 , which does not appear to be associated with any eigenvalue computed by CMASS_VAX. Differences two through five were found to be the result of the differences in the state matrices computed by CMASS and CMASS_VAX (see below). Thus, the significance of these differences depends on whether the T3500 state matrices computed by CMASS and by CMASS_VAX are different because of roundoff errors or because of an error in CMASS. This is an additional reason to determine the reason why the T3500 state matrices computed by CMASS and by CMASS_VAX are different.

It was verified that eigenvalue differences two through five were the result of differences in the T3500 state matrices by 1) printing the $T 3500$ state matrix computed by CMASS_VAX to a file, 2) modifying CMASS to read in a state matrix, and 3 ) using the modified version of CMASS to compute the eigenvalues of the VAX-generated state matrix. The eigenvalues computed by the modified version of CMASS are nearly identical to the eigenvalues computed by CMASS_VAX, which shows that differences two through five are the result of the differences in the state matrices computed by CMASS and CMASS_VAX.

The tests described in this appendix have demonstrated that 1) CMASS_VAX and the original VAX version of MASS, MASSV2-4K, yield essentially the same results and 2) most of the code in CMASS works correctly. The tests in this appendix, however, raise the question: What is the cause of the differences in the state matrices computed by CMASS and by CMASS_VAX for the T3500 problem? This question should be answered before CMASS is used for the analysis of power systems. Furthermore, the six test problems supplied with MASS do not test all of the options in MASS. Hence, more extensive testing of MASS should be performed before using CMASS for the analysis of power systems. 
APPENDIX C

A 2200-STATE CRAY VERSION OF MASS 
APPENDIX C

\section{A 2200-STATE CRAY VERSION OF MASS}

A 2200-state version of CMASS, CMASS_2200, was generated by replacing the files CMASS.FOR and CMASS_INV.FOR (see Appendix $B$ ) with the files CMASS_2200.FOR and CMASS_INY22.FOR, respectively. The differences between these files are described in this appendix.

CMASS_INV22.FOR is the same as CMASS_INV.FOR, except that a global substitution was done to change the parameters NDAT $=9000, N M=500, M P=250$, $N G=250, N Z L=500$, NDEV $=300$, and $N S I=500$ to $N D A T=15000, N M=900, M P=300, N G=300$, $N Z L=1500$, NDEV $=900$, and NSI $=2200$. This change makes the arrays in CMASS_2200 large enough to run the $\mathrm{BCH}_{88 \mathrm{HW}} \mathrm{W}_{2}$ and $\mathrm{BCH}_{88 \mathrm{HW}} \mathrm{H}_{3} 3$ problems.

The first difference between CMASS.FOR and CMASS_2200.FOR is that a global substitution was done to change the parameters NDAT $=9000, N M=500$, $M P=250, N G=250, N Z L=500$, NDEV $=300$, and $N S I=500$ to $N D A T=15000, N M=900, M P=300$, $N G=300, N Z L=1500, N D E V=900$, and $N S I=2200$. This change makes the arrays in CMASS_2200 large enough to run the BCH88HW3_2 and BCH88HW3_3 problems.

The second difference between CMASS.FOR and CMASS_2200.FOR is that the line

IF (KGROUP.NE.0) CALL MRESP

in subroutine FMODE (i.e., line 22651 in CMASS. FOR), was replaced by C**DEL

C* IF(KGROUP.NE.0) CALL MRESP

$C \star \star D E L$ END.

This allows subroutines MRESP, DPLT and DFPLT to be deleted.

The third difference between CMASS.FOR and CMASS_2200.FOR is that the line

IF (JF.LT.NSTATE)GOTO 1230

in subroutine AEIGN (i.e., line 27172 in CMASS.FOR) was replaced by 


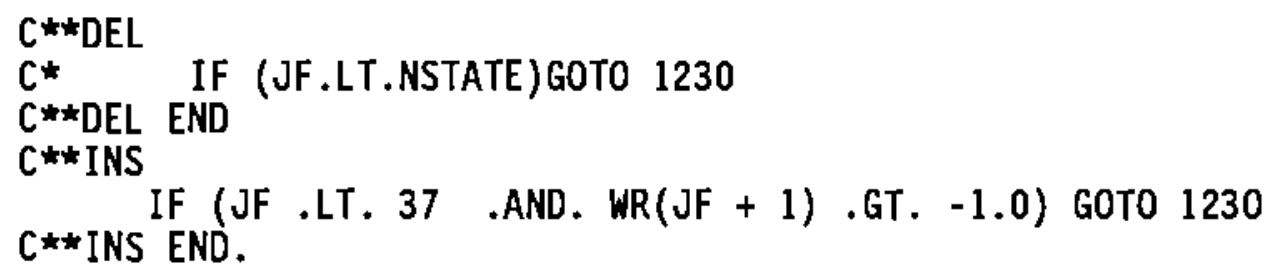

This modification causes CMASS_2200 to print a participation vector or eigenvector only if the real part of the corresponding eigenvalue is greater than -1.0 , and it allows a maximum of only about 37 vectors to be printed. This allows some participation vectors and right eigenvectors to be printed without having an excessively large output file for large problems.

The fourth difference between CMASS.FOR and CMASS_2200.FOR is that the line

2180 FORMAT $\left(1 X,{ }^{\prime} X^{\prime}, 13,15,1 X, 3 A 4,1 X, A 4, A 4,10(F 10.3)\right)$

in subroutine AEIGN (i.e., line 27245 in CMASS. FOR) was replaced by

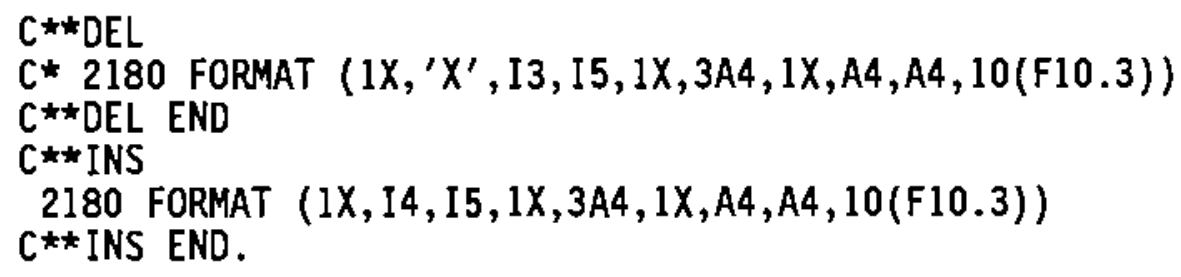

This allow CMASS_2200 to correctly output state variable numbers when there are more than 999 states.

The fifth, and last, difference between CMASS.FOR and CMASS_2200.FOR is that subroutines MRESP, DPLT, and DFPLT were deleted. This eliminates an array requiring $\mathrm{N}^{2}$ words of memory.

The third modification does not give the desired result if IPRNT is equal to one (1) or minus one $(-1)$ in the PRINT SPECIFICATION AND CHANGE CASE data file. In particular, CMASS_2200 will not output results as expected if the user requests that the participation vectors for unstable eigenvalues and for eigenvalues associated with rotor angle or speed be printed.

It was verified that CMASS_2200 works correctly by running the 11BUS and T3500 test problems using CMASS_2200. Using the Cray differences utility, it was found that CMASS and CMASS_2200 produced essentially identical results 
for these problems. A11 of the differences between the outputs of CMASS_2200 and CMASS are consistent with the differences between CMASS.FOR and CMASS_2200.FOR. 



\section{DISTRIBUTION}

No. of

Copies

\section{OFFSITE}

2 DOE/Office of Scientific and Technical Information

4 BPA Library - SSL

Bonneville Power Administration P.0. Box 3621

Portland, OR 97208

5 Bonneville Power Administration ATTN: D. McNulty - EOHB

P.0. Box 3621

Portland, OR 97208

H. M. Harwick

Battel le Portland Operations

Suite 650

500 NE Multnomah

Portland, OR 97232
No. of

Copjes

\section{ONSITE}

DOE Richland Operations Office

R. B. Goranson

20 Pacific Northwest Laboratory

J. G. DeSteese

D. M. Elwood (10)

P. S. Lowery

E. W. Pearson

G. L. Work

Publishing Coordination

Technical Report Files (5) 
